\title{
EMBEDDINGS OF THE COMPLEX BALL INTO SIEGEL SPACE *
}

\author{
OLIVER BÜLTEL ${ }^{\dagger}$
}

Abstract. We study properties of a certain map from the unitary group $U(1, n-1)$ to the group $\mathrm{U}\left(\left(\begin{array}{c}n-1 \\ k-1\end{array}\right),\left(\begin{array}{c}n-1 \\ k\end{array}\right)\right)$. We explain how it gives rise to a map between canonical models of Shimura varieties and we prove that it extends to the ordinary locus of the integral model. Finally, we extend results of Satake on the endomorphism ring of a generic image point to positive characteristics.

1. Introduction. In [14] Satake classifies embeddings of a symmetric Hermitian space $X$ into Siegel space, let us describe his results in a special case: The bounded realization of $I_{p, n-p}$ is a domain in complex affine $p(n-p)$-space which may conveniently be described as the set of complex valued $p \times(n-p)$-matrices

$$
B_{p, n-p}=\left\{A \in \operatorname{Mat}(p, n-p) \mid\|A\|_{\infty}<1\right\}
$$

where $\|\cdot\|_{\infty}$ is the operatornorm (relative to the standard Hilbert space norms $\|\cdot\|_{2}$ on $\mathbb{C}^{p}$ and $\mathbb{C}^{n-p}$ respectively). If we write $S_{n}$ for the bounded realization of $I I I_{n}$, then there is a natural holomorphic embedding

$$
B_{p, n-p} \hookrightarrow S_{n} .
$$

In the special case $p=1$ we recover the complex ball in $\mathbb{C}^{n-1}$. Surprisingly (1.1) is not the sole symplectic embedding of $B_{1, n-1}$, as one has maps

$$
B_{1, n-1} \hookrightarrow B_{\left(\begin{array}{c}
n-1 \\
k-1
\end{array}\right),\left(\begin{array}{c}
n-1 \\
k
\end{array}\right)} \hookrightarrow S_{\left(\begin{array}{c}
n \\
k
\end{array}\right)}
$$

which are induced by sending $x \in \mathbb{C}^{n-1}$ to the $\left(\begin{array}{c}n-1 \\ k-1\end{array}\right) \times\left(\begin{array}{c}n-1 \\ k\end{array}\right)$-matrix $A$ with entries

$$
A_{I, J}= \begin{cases}(-1)^{\nu-1} x_{i_{\nu}} & I=J-\left\{i_{\nu}\right\} \\ 0 & \text { otherwise }\end{cases}
$$

where $I=\left\{i_{1}, \ldots, i_{k-1}\right\}$ and $J=\left\{i_{1}, \ldots, i_{k}\right\}$ run through all subsets of $\{1, \ldots, n-1\}$ with cardinalities $k-1$ and $k$. We want to study the effect of (1.2) to quotients of $B_{1, n-1}$ by congruence subgroups and their canonical models. We consider, in more modern language, a Shimura datum $(G, X)$, such that $G$ is a $\mathbb{Q}$-form of $\operatorname{GL}(n, \mathbb{C})$ and such that the conjugacy class $\mu$ of minuscule cocharacters obtained from $X$ is:

$$
\mathbb{C}^{\times} \ni z \mapsto\left(\begin{array}{cccc}
z & 0 & \ldots & 0 \\
0 & 1 & \ldots & 0 \\
\vdots & \vdots & \ddots & \vdots \\
0 & 0 & \ldots & 1
\end{array}\right) \in \mathrm{GL}(n, \mathbb{C})
$$

If $\mu$ is as above then all of the $n-1$ fundamental dominant weights $\lambda$ satisfy Deligne's condition $\langle\mu, \lambda+c(\lambda)\rangle=1, c$ is the Weil-opposition. Thus one expects by $[2,1.3]$ that (suitable $\mathbb{Q}$ forms of) their highest weight representations provide maps from the Shimura datum $(G, X)$ to a symplectic Shimura datum $\left(\operatorname{GSp}\left(2\left(\begin{array}{l}n \\ k\end{array}\right), \mathbb{Q}\right), S_{\left(\begin{array}{c}n \\ k\end{array}\right)}^{ \pm}\right)$. Such

*Received November 24, 2002; accepted for publication May 9, 2003.

${ }^{\dagger}$ Mathematisches Institut der Universität Heidelberg, Im Neuenheimer Feld 288, 69120 Heidelberg, Germany (bueltel@mathi.uni-heidelberg.de). 
a symplectic representation of $(G, X)$ gives rise to the map (1.2) of the underlying symmetric Hermitian space, which in our case is the complex ball. Now consider a neat level structure $K \subset G\left(\mathbb{A}^{\infty}\right)$. By Deligne's theory we obtain a Shimura variety

$$
M(\mathbb{C})=G(\mathbb{Q}) \backslash\left(X \times G\left(\mathbb{A}^{\infty}\right) / K\right)
$$

which has weakly canonical models over all fields containing the reflex. Moreover, the aforementioned symplectic maps provide us with certain abelian schemes $Y^{(k)}$ over $M$. (Here one has to allow to replace $(G, X)$ by a certain covering Shimura datum denoted $\left(G_{1}, X_{1}\right) \rightarrow(G, X)$ in [2, Proposition 2.3.10], see body of text for details.) If one assumes that $G$ and $K$ are sufficiently well behaved at $p$, then the theory of $P E L$-moduli spaces provides us with a smooth model $\mathcal{M}$.

In this paper we study the extension properties of $Y^{(k)}$ with respect to the model $\mathcal{M}$. Our result is that $Y^{(k)}$ extends to an abelian scheme at least over the ordinary locus of $\mathcal{M}$. Our result holds for all primes including 2 .

In a future publication we will show that one can remove the ordinarity hypothesis at the cost of having to restrict to primes bigger than $k+1$ along with somewhat stronger conditions on $G$ and $K$. More general results (under similar restrictions on the prime) have already been shown in [18].

The work is organised as follows: In section 2 we describe the Shimura datum $\left(G_{1}, X_{1}\right)$ and the abelian schemes $Y^{(k)}$ using the language of Hodge structures, we do not attempt to work with all possible $\mathbb{Q}$-forms of $G$ and of $G_{1}$ but rather confine ourself to what we think is the most natural one. This allows us to give a very down to earth (and we hope enlightening) treatment of the covering Shimura datum $\left(G_{1}, X_{1}\right)$ (denoted $\left(G^{(0 \times 1)}, X^{(0 \times 1)}\right)$ in body of text), and of its symplectic representations (which we denote by $V^{(k)}$ ). In section 3 we recall canonical coordinates and canonical lifts, to a large extend with sketchy proof. In section 4 we show that under the usual assumptions on $K$ the variety $M$ has a smooth model over the ring of integers in the local field $E_{\mathfrak{p}}$, where $E$ is a certain finite extension of the reflex field and where $\mathfrak{p}$ is a prime over $p$. In section 5 we prove the extension theorem in two steps, we start with the construction of an extension of the Barsotti-Tate group of $Y^{(k)}$, and then we show that this determines a well-defined extension of $Y^{(k)}$ itself, essentially by Zariski's main theorem. Finally, we show in section 6 how some of Satake's work on the endomorphism ring of $Y^{(k)}$ can be carried over to the points in the ordinary locus of the special fibre of $\mathcal{M}$.

I thank Prof. Ivan Fesenko, Prof. Eberhard Freitag, Prof. Winfried Kohnen, Prof. Richard Pink, Prof. Richard Taylor, and Prof. Rainer Weissauer for interesting conversations on the topic and further thanks go to the referee.

2. Exterior powers of Hodge structures. Let $V$ be a finitely generated tor-

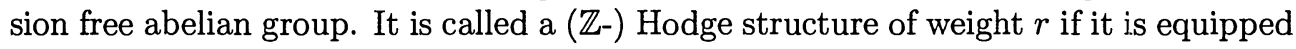
with a decomposition

$$
V_{\mathbb{C}} \cong \bigoplus_{p+q=r} V^{p, q}
$$

satisfying $V^{q, p}=\overline{V^{p, q}}$. A direct sum

$$
V \cong \bigoplus_{r} V_{r}
$$




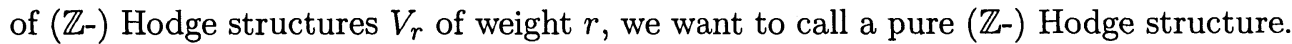
To give the decomposition over the reals is equivalent to give a homomorphism of $\mathbb{R}$-algebraic groups

$$
h: \mathbb{C}^{\times} \rightarrow \mathrm{GL}_{\mathbb{R}}\left(V_{\mathbb{R}}\right) \subset \mathrm{GL}_{\mathbb{C}}\left(V_{\mathbb{C}}\right)
$$

by making $z \in \mathbb{C}^{\times}$act on $V^{p, q}$ by $z^{-p} \bar{z}^{-q}$. If the only non-zero direct summands amongst the $V^{p, q}$ 's are $V^{-1,0}$ and $V^{0,-1}$ then the homomorphism above corresponds to a homomorphism between the $\mathbb{R}$-algebras $\mathbb{C}$ and $\operatorname{End}_{\mathbb{R}}\left(V_{\mathbb{R}}\right)$, which we continue to denote by $h$. In that case $V$ is called a Hodge structure of type $\{(-1,0),(0,-1)\}$, and the $\mathbb{C}$-space $V^{-1,0}$ can set-theoretically be identified with $V_{\mathbb{R}}$ which acquires its $\mathbb{C}$-vector space structure by the corresponding algebra homomorphism $h: \mathbb{C} \rightarrow$ $\operatorname{End}_{\mathbb{R}}\left(V_{\mathbb{R}}\right)$. Pure Hodge structures form an additive $\otimes$-category in an obvious way. The pure Hodge structures of type $\{(-1,0),(0,-1)\}$ are an additive full subcategory thereof.

Let $\mathcal{O}_{L}$ be the ring of integers in a totally imaginary quadratic extension $L$ of a totally real number field $L^{+}$. Write $*$ for the non-trivial involution of $L / L^{+}$. A Hodge structure $V$ is said to have a $\mathcal{O}_{L}$-operation if a homomorphism $\iota: \mathcal{O}_{L} \rightarrow \operatorname{End}(V)$ is given. If $\iota$ is fixed we obtain a refinement of the Hodge decomposition:

$$
V_{\sigma}=V \otimes_{L, \sigma} \mathbb{C} \cong \bigoplus_{p, q} V_{\sigma}^{p, q}
$$

where we denote for any embedding $\sigma: L \rightarrow \mathbb{C}$ by $V_{\sigma}^{p, q}$ the $\sigma$-eigenspace of $V^{p, q}$. Note that we have $V_{\sigma}^{q, p} \cong \overline{V_{\sigma \circ *}^{p, q}}$. The Hodge structures with $\mathcal{O}_{L^{-}}$-operation form a $\otimes$-category as follows: If $V$ and $W$ are given, we can form the finitely generated torsion free $\mathcal{O}_{L}$-module $V \otimes_{\mathcal{O}_{L}} W$. We make it into a Hodge structure by declaring the subspace of $V_{\sigma} \otimes_{\mathbb{C}} W_{\sigma}$ of weight $(p, q)$ to be:

$$
\bigoplus_{p_{1}, q_{1}} V_{\sigma}^{p_{1}, q_{1}} \otimes_{\mathbb{C}} W_{\sigma}^{p-p_{1}, q-q_{1}}
$$

In a similar way we define $\bigwedge_{\mathcal{O}_{L}}^{k} V$. We want to describe a particularly interesting example of such tensor constructions, to this end fix a natural number $n$. Fix two different $C M$-traces $\Phi^{(0)}, \Phi^{(n)}: L \rightarrow \mathbb{C}\left(=\mathbb{Q}\right.$-linear maps which arise as $\Phi^{(k)}(x)=$ $\sum_{\sigma \in\left|\Phi^{(k)}\right|} \sigma(x)$ for all $x \in L$ and $k \in\{0, n\}$, where $\left.\left|\Phi^{(0)}\right|,\left|\Phi^{(n)}\right| \subset \operatorname{Hom}_{\mathbb{Q}}(L, \mathbb{C})\right)$. Let us define:

$$
\Phi^{(1)}(x)=(n-1) \Phi^{(0)}(x)+\Phi^{(n)}(x)
$$

Our input is a choice of:

- two Hodge structures of type $\{(-1,0),(0,-1)\}$ with $\mathcal{O}_{L}$-operation, i.e. $\mathbb{R}$ linear homomorphisms

$$
h^{(k)}: \mathbb{C} \rightarrow \operatorname{End}_{L}\left(V_{\mathbb{R}}^{(k)}\right),
$$

for $k \in\{0,1\}$, where the $V^{(k)}$ are finitely generated, torsion free $\mathcal{O}_{L}$-modules. We require that:

$$
\operatorname{tr}\left(\left.x\right|_{V^{(k)-1,0}}\right)=\Phi^{(k)}(x),
$$

hold for all $x \in L$, and $k \in\{0,1\}$. 
- two *-skew-Hermitian $\mathbb{Q}$-valued forms $\psi^{(1)}$, and $\psi^{(0)}$ on the $L$-spaces $V_{\mathbb{Q}}^{(1)}$, and $V_{\mathbb{Q}}^{(0)}$, such that firstly the $h^{(k)}$ become $*$-involution preserving homomorphisms, if the right hand side of (2.2) is endowed with the Rosati involution, and such that secondly the forms $\psi^{(k)}\left(x, h^{(k)}(i) y\right)$ on $V_{\mathbb{R}}^{(k)}$ are positive definite.

Observe that (2.3) implies that the $\mathcal{O}_{L}$-modules $V^{(1)}$ and $V^{(0)}$ have the ranks $n$ and 1. Observe also that we may write the forms $\psi^{(1)}$ and $\psi^{(0)}$ in a unique way as traces $\operatorname{tr}_{L / \mathbb{Q}} \Psi^{(1)}$, and $\operatorname{tr}_{L / \mathbb{Q}} \Psi^{(0)}$, where $\Psi^{(1)}$, and $\Psi^{(0)}$ are sesquilinear forms that are $L$-valued on $V_{\mathbb{Q}}^{(1)}$, and on $V_{\mathbb{Q}}^{(0)}$. Based on the 2 nd and 3 rd row of the table on page 188 of [14, Chapter IV,Paragraph 5] we want to consider the following output:

1. $\mathcal{O}_{L}$-modules $V^{(k)}$ defined by:

$$
V^{(0)^{\otimes \mathcal{O}_{L}}{ }^{1-k}} \otimes_{\mathcal{O}_{L}} \bigwedge_{\mathcal{O}_{L}}^{k} V^{(1)},
$$

for every $k \in\{0, \ldots, n\}$,

2. $\mathbb{Q}$-valued $(L, *)$-skew-Hermitian forms $\psi^{(k)}=\operatorname{tr}_{L / \mathbb{Q}} \Psi^{(k)}$ on $V_{\mathbb{Q}}^{(k)}$, here is:

$$
\begin{gathered}
\Psi^{(k)}\left(x_{0}^{1-k} x_{1} \wedge \cdots \wedge x_{k}, y_{0}^{1-k} y_{1} \wedge \cdots \wedge y_{k}\right) \\
=\left(\Psi^{(0)}\left(x_{0}, y_{0}\right)\right)^{1-k} \operatorname{det}\left(\Psi^{(1)}\left(x_{i}, y_{j}\right)_{i, j}\right)
\end{gathered}
$$

for $x_{1}, \ldots, x_{k}, y_{1}, \ldots, y_{k} \in V_{\mathbb{Q}}^{(1)}$, and $x_{0}, y_{0} \in V_{\mathbb{Q}}^{(0)}$,

3. *-homomorphisms $h^{(k)}: \mathbb{C}^{\times} \rightarrow \operatorname{Aut}\left(V_{\mathbb{R}}^{(k)}\right)$ defined so that $z \in \mathbb{C}^{\times}$acts as:

$$
x_{0}^{1-k} x_{1} \wedge \cdots \wedge x_{k} \mapsto\left(h^{(0)}(z) x_{0}\right)^{1-k} h^{(1)}(z) x_{1} \wedge \cdots \wedge h^{(1)}(z) x_{k} .
$$

We have to introduce reductive $\mathbb{Q}$-groups as follows, on the category of all $\mathbb{Q}$-algebras $G^{(k)}$ represents the functor:

$$
C \mapsto\left\{(\gamma, \mu) \in \operatorname{End}_{L \otimes C}^{\times}\left(V_{C}^{(k)}\right) \times C^{\times} \mid \psi^{(k)}(\gamma x, \gamma y)=\mu \psi^{(k)}(x, y)\right\}
$$

Notice the natural similitude morphisms from $G^{(k)}$ to $\mathbb{G}_{m}$. We also introduce a group $G^{(0 \times 1)}$ by the requirement that the diagram

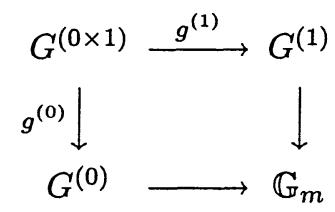

be cartesian. Finally we introduce $\mathbb{Q}$-rational group homomorphisms $g^{(k)}: G^{(0 \times 1)} \rightarrow$ $G^{(k)}$ by sending, say $\left(\gamma^{(0)}, \gamma^{(1)}\right) \in G^{(0 \times 1)}(C)$ to the element $\gamma^{(k)}: V_{C}^{(k)} \rightarrow V_{C}^{(k)}$ defined by

$$
\gamma^{(k)}\left(x_{0}^{1-k} x_{1} \wedge \cdots \wedge x_{k}\right)=\gamma^{(0)}\left(x_{0}\right)^{1-k} \gamma^{(1)}\left(x_{1}\right) \wedge \cdots \wedge \gamma^{(1)}\left(x_{k}\right)
$$

Let us also pick connected, smooth $\mathbb{Z}$-models $G_{\mathbb{Z}}^{(k)}\left(G_{\mathbb{Z}}^{(0 \times 1)}\right)$ of the groups $G^{(k)}\left(G^{(0 \times 1)}\right)$ by taking the schematic closure in the group $\mathbb{Z}$-schemes $\operatorname{GL}\left(V^{(k)} / \mathbb{Z}\right)\left(\mathrm{GL}\left(V^{(0)} \oplus\right.\right.$ 
$\left.\left.V^{(1)} / \mathbb{Z}\right)\right)$. Here the $\mathcal{O}_{L}$-lattices $V^{(k)} \subset V_{\mathbb{Q}}^{(k)}\left(V^{(0)} \oplus V^{(1)} \subset V_{\mathbb{Q}}^{(0)} \oplus V_{\mathbb{Q}}^{(1)}\right)$, are as before. The maps $g^{(k)}$ preserve these lattices and so give rise to maps $g_{\mathbb{Z}}^{(k)}: G_{\mathbb{Z}}^{(0 \times 1)} \rightarrow G_{\mathbb{Z}}^{(k)}$. The $V^{(k)}$ are clearly Hodge structures with $\mathcal{O}_{L}$-operation, however much more is true:

Lemma 2.1. Let $\left(V^{(1)}, V^{(0)}, \psi^{(1)}, \psi^{(0)}, h^{(1)}, h^{(0)}\right)$ be as before. Then $\left(V^{(k)}, h^{(k)}\right)$ is a Hodge structure of type $\{(-1,0),(0,-1)\}$ with $\mathcal{O}_{L}$-operation. Moreover, the quadruples

$$
\left(L, *, V_{\mathbb{Q}}^{(k)}, \psi^{(k)}, h^{(k)}\right)
$$

are PEL-data in the sense of [8], as is the quadruple

$$
\left(L \oplus L, *, V_{\mathbb{Q}}^{(0)} \oplus V_{\mathbb{Q}}^{(1)}, \psi^{(0)} \oplus \psi^{(1)}, h^{(0)} \oplus h^{(1)}\right) .
$$

The $G^{(k)}(\mathbb{R})$-conjugacy class of $h^{(k)}$ is determined by the formula:

$$
\operatorname{tr}\left(\left.x\right|_{V^{(k)-1,0}}\right)=\Phi^{(k)}(x)=\left(\begin{array}{c}
n-1 \\
k
\end{array}\right) \Phi^{(0)}(x)+\left(\begin{array}{c}
n-1 \\
k-1
\end{array}\right) \Phi^{(n)}(x) .
$$

for any $x \in L$. Therefore, when writing $E^{\delta}$ for the reflex field of $\left(G^{\delta}, h^{\delta}\right)$, we have $E^{(k)} \subset E^{(0 \times 1)}$ for all $k$. Finally, the map $g^{(k)}: G^{(0 \times 1)} \rightarrow G^{(k)}$ takes $h^{(0 \times 1)}:=$ $h^{(0)} \oplus h^{(1)}$ to $h^{(k)}$.

Proof. We first check that the Hodge structure $V^{(k)}$ has weights in the set $\{(-1,0),(0,-1)\}$. The assumption (2.3) on $V^{(1)}$ implies that the $V_{\sigma}^{(1)^{-1,0}}$ have the following dimensions, where $\sigma: L \rightarrow \mathbb{C}$ runs through all embeddings:

$$
\operatorname{dim}_{\mathbb{C}} V_{\sigma}^{(1)^{-1,0}}= \begin{cases}n & \text { if } \sigma \in\left|\Phi^{(0)}\right| \cap\left|\Phi^{(n)}\right| \\ n-1 & \text { if } \sigma \in\left|\Phi^{(0)}\right|-\left|\Phi^{(n)}\right| \\ 1 & \text { if } \sigma \in\left|\Phi^{(n)}\right|-\left|\Phi^{(0)}\right| \\ 0 & \text { if } \sigma \notin\left|\Phi^{(0)}\right| \cup\left|\Phi^{(n)}\right|\end{cases}
$$

and analogous formulas for $\operatorname{dim}_{\mathbb{C}} V_{\sigma}^{(1)^{0,-1}}$ hold. Consequently we derive the following Hodge decomposition for the $\sigma$-eigenspace of $\bigwedge_{\mathcal{O}_{L}}^{k} V^{(1)}$ :

$$
\bigwedge_{\mathbb{C}}^{k} V_{\sigma}^{(1)}= \begin{cases}\bigwedge_{\mathbb{C}}^{k} V_{\sigma}^{(1)^{-1,0}} & \text { if } \sigma \in\left|\Phi^{(0)}\right| \cap\left|\Phi^{(n)}\right| \\ \bigwedge_{\mathbb{C}}^{k} V_{\sigma}^{(1)^{-1,0}} \oplus \bigwedge_{\mathbb{C}}^{k-1} V_{\sigma}^{(1)^{-1,0}} \otimes_{\mathbb{C}} V_{\sigma}^{(1)^{0,-1}} & \text { if } \sigma \in\left|\Phi^{(0)}\right|-\left|\Phi^{(n)}\right| \\ V_{\sigma}^{(1)^{-1,0}} \otimes_{\mathbb{C}} \bigwedge_{\mathbb{C}}^{k-1} V_{\sigma}^{(1)^{0,-1}} \oplus \bigwedge_{\mathbb{C}}^{k} V_{\sigma}^{(1)^{0,-1}} & \text { if } \sigma \in\left|\Phi^{(n)}\right|-\left|\Phi^{(0)}\right| \\ \bigwedge_{\mathbb{C}}^{k} V_{\sigma}^{(1)^{0,-1}} & \text { if } \sigma \notin\left|\Phi^{(0)}\right| \cup\left|\Phi^{(n)}\right|\end{cases}
$$

so that in each of the four cases the $\sigma$-eigenspace of $\bigwedge_{\mathcal{O}_{L}}^{k} V^{(1)}$ has the following Hodge weights (with multiplicity):

$$
(p, q)= \begin{cases}\left(\begin{array}{l}
n \\
k
\end{array}\right) \times(-k, 0) & \text { if } \sigma \in\left|\Phi^{(0)}\right| \cap\left|\Phi^{(n)}\right| \\
\left(\begin{array}{c}
n-1 \\
k
\end{array}\right) \times(-k, 0),\left(\begin{array}{c}
n-1 \\
k-1
\end{array}\right) \times(1-k,-1) & \text { if } \sigma \in\left|\Phi^{(0)}\right|-\left|\Phi^{(n)}\right| \\
\left(\begin{array}{c}
n-1 \\
k-1
\end{array}\right) \times(-1,1-k),\left(\begin{array}{c}
n-1 \\
k
\end{array}\right) \times(0,-k) & \text { if } \sigma \in\left|\Phi^{(n)}\right|-\left|\Phi^{(0)}\right| \\
\left(\begin{array}{l}
n \\
k
\end{array}\right) \times(0,-k) & \text { if } \sigma \notin\left|\Phi^{(0)}\right| \cup\left|\Phi^{(n)}\right|\end{cases}
$$


Comparing this with the Hodge weights of $V_{\sigma}^{(0)^{\otimes \mathbb{C} 1-k}}$, being

$$
(p, q)= \begin{cases}(k-1,0) & \text { if } \sigma \in\left|\Phi^{(0)}\right| \\ (0, k-1) & \text { if } \sigma \notin\left|\Phi^{(0)}\right|\end{cases}
$$

shows that the sole weights of $V^{(k)}$ are $\{(-1,0),(0,-1)\}$ and shows the formula (2.5) also.

We still have to verify that the pairings $\psi^{(k)}\left(x, h^{(k)}(i) y\right)$ are positive definite. Every embedding $\sigma: L \rightarrow \mathbb{C}$ is a $*$-homomorphism, therefore one obtains sesquilinear $\mathbb{C}$ valued pairings $\Psi_{\sigma}^{(k)}$ on $V_{\sigma}^{(k)}$ for every $k$. Consider the forms $E_{\sigma}^{(k)}=\Psi_{\sigma}^{(k)}\left(x, h^{(k)}(i) y\right)$. By assumption $\psi^{(1)}\left(x, h^{(1)}(i) y\right)$, and $\psi^{(0)}\left(x, h^{(0)}(i) y\right)$ are positive definite on $V_{\mathbb{R}}^{(1)}$, and $V_{\mathbb{R}}^{(0)}$, so these forms restrict to positive definite forms $E_{\sigma}^{(1)}$, and $E_{\sigma}^{(0)}$ on $V_{\sigma}^{(1)}$, and $V_{\sigma}^{(0)}$. By utilizing the formulas for $\Psi^{(k)}$ and $h^{(k)}$ we see the positive definiteness of $E_{\sigma}^{(k)}$, as

$$
E_{\sigma}^{(k)}\left(x_{0}^{1-k} x_{1} \wedge \cdots \wedge x_{k}, y_{0}^{1-k} y_{1} \wedge \cdots \wedge y_{k}\right)=E_{\sigma}^{(0)}\left(x_{0}, y_{0}\right)^{1-k} \operatorname{det}\left(E_{\sigma}^{(1)}\left(x_{i}, y_{j}\right)_{i, j}\right) .
$$

Finally just check that $\psi^{(k)}\left(x, h^{(k)}(i) y\right)$ is the sum of various $E_{\sigma}^{(k)}(x, y)$ 's.

We turn to Shimura varieties which correspond to our choice of $V_{\mathbb{Q}}^{\delta}, \psi^{\delta}, h^{\delta}$, for $\delta \in\{(0 \times 1),(0), \ldots,(n)\}$. Let $X^{\delta}$ be the conjugacy class of $h^{\delta}$ in $G_{\mathbb{R}}^{\delta}$, (we set $\left.h^{(0 \times 1)}=h^{(0)} \oplus h^{(1)}\right)$, then one puts according to $[1]$ :

$$
{ }_{K} M_{\mathbb{C}}\left(G^{\delta}, X^{\delta}\right)=G^{\delta}(\mathbb{Q}) \backslash\left(X^{\delta} \times G^{\delta}\left(\mathbb{A}^{\infty}\right) / K\right)
$$

for the Shimura varieties, of which the projective limit $\lim _{K \rightarrow 1} K M_{\mathbb{C}}\left(G^{\delta}, X^{\delta}\right)$ is equal to

$$
M_{\mathbb{C}}\left(G^{\delta}, X^{\delta}\right)=G^{\delta}(\mathbb{Q}) \backslash\left(X^{\delta} \times G^{\delta}\left(\mathbb{A}^{\infty}\right)\right),
$$

by [2, Corollaire 2.1.11]. One writes ${ }_{K} M\left(G^{\delta}, X^{\delta}\right)$ and $M\left(G^{\delta}, X^{\delta}\right)$, for their weakly canonical models over a choice of any field containing the reflex. We will prefer to always work over the field $E^{(0 \times 1)}$, being the largest of the reflex fields $E^{\delta}$, thus our $M\left(G^{(k)}, X^{(k)}\right)$ 's are strictly speaking base changes via $\times_{E^{(k)}} E^{(0 \times 1)}$ of the canonical models.

Let us briefly sketch the moduli interpretations due to Deligne [1, Scholie 4.11]. If $F / E^{(0 \times 1)}$ is an algebraically closed field then there is a $\operatorname{Aut}_{E^{(0 \times 1)}}(F) \times G^{(k)}\left(\mathbb{A}^{\infty}\right)$ equivariant bijection between $F$-valued points of $M\left(G^{(k)}, X^{(k)}\right)$ and isogeny classes of quadruples $\left(Y^{(k)}, \iota^{(k)}, \lambda^{(k)}, \eta^{(k)}\right)$ such that:

(a) $Y^{(k)}$ is an abelian variety over $F$ up to isogeny.

(b) $\iota^{(k)}: L \rightarrow \operatorname{End}^{0}\left(Y^{(k)}\right)$ is a homomorphism such that

$$
\operatorname{tr}_{\operatorname{Lie} Y^{(k)}}\left(\iota^{(k)}(x)\right)=\Phi^{(k)}(x)
$$

for all $x \in L$.

(c) $\lambda^{(k)}$ is a homogeneous polarization of $Y^{(k)}$ of which the Rosati involution restricts to the $C M$ involution on $L$.

(d) a $L$-linear level structure $\eta^{(k)}: V_{\mathbb{A}}^{\infty(k)} \rightarrow H_{1}\left(Y^{(k)}, \mathbb{A}^{\infty}\right)$ which becomes a symplectic similitude if one imposes the Weil pairing on the right and the pairing $\psi^{(k)}$ on the left.

(e) the skew-Hermitian $L$-module $H_{1}\left(Y^{(k)} \times_{F} \mathbb{C}, \mathbb{Q}\right)$ is isomorphic to $\left(V_{\mathbb{Q}}^{(k)}, \psi^{(k)}\right)$ 
and similarly for $M\left(G^{(0 \times 1)}, X^{(0 \times 1)}\right)$. In fact a more thorough treatment can be found in [8]: The scheme $M\left(G^{(0 \times 1)}, X^{(0 \times 1)}\right)$ represents a functor taking a $E^{(0 \times 1)}$-scheme $S$ to the set of tuples $\left(Y^{(k)}, \iota^{(k)}, \lambda^{(k)}, \eta^{(k)}\right)$ with properties analogous to (a)-(e). From now on let us pick a level $l$ which is $\geq 3$. The compact open groups

$$
K^{\delta}=\left\{\gamma \in G_{\mathbb{Z}}^{\delta}(\hat{\mathbb{Z}}) \mid \gamma \equiv 1 \quad(\bmod l)\right\}
$$

are neat and satisfy $g^{(k)}\left(K^{(k)}\right) \subset K^{(0 \times 1)}$. This is most useful as it sets up maps between the characteristic zero Shimura varieties

$$
g^{(k)}:_{K^{(0 \times 1)}} M\left(G^{(0 \times 1)}, X^{(0 \times 1)}\right) \rightarrow K^{(k)} M\left(G^{(k)}, X^{(k)}\right)
$$

that are induced from the group theoretic maps of lemma 2.1, according to $[1$, Corollaire 5.4]. Note that these maps do not have a natural moduli interpretation. Nevertheless we do obtain a homogeneously polarized abelian scheme $Y^{(k)}$ up to isogeny over $K^{(0 \times 1)} M\left(G^{(0 \times 1)}, X^{(0 \times 1)}\right)$, with $\mathcal{O}_{L}$-operation $\iota^{(k)}$, and appropriate level structure $\eta^{(k)}$, by pulling back the universal family on $K^{(k)} M\left(G^{(k)}, X^{(k)}\right)$ via $g^{(k)}$. Here, we wish to consider the particular representative of the isogeny class $Y^{(k)}$ which is determined by the constraint $\eta^{(k)}\left(V_{\hat{\mathbb{Z}}}^{(k)}\right)=H_{1}\left(Y_{\xi}^{(k)}, \hat{\mathbb{Z}}\right)$. Within the homogeneous class of polarizations $\lambda^{(k)}$, coming from the data $\mathbb{Q} \psi^{(k)}$, we wish to pick some effective representative and write $d_{k}$ for its degree. Note that the homogeneous class $\mathbb{Q} \psi^{(k)}$ depends only on the homogeneous class $\mathbb{Q}\left(\psi^{(0)} \oplus \psi^{(1)}\right)$ of polarizations on $V^{(0)} \oplus V^{(1)}$, but the choice of an effective representative is arbitrary. Note also that $\psi^{(k)}$ may well be ineffective even if $\psi^{(0)} \oplus \psi^{(1)}$ is effective, see lemma 5.1 below, however. Having made the above choice we obtain a further map:

$$
g^{(k)}:{ }_{K^{(0 \times 1)}} M\left(G^{(0 \times 1)}, X^{(0 \times 1)}\right) \rightarrow \mathcal{A}_{g_{k}, d_{k}, l}
$$

where $g_{k}$ is $\left[L^{+}: \mathbb{Q}\right]\left(\begin{array}{l}n \\ k\end{array}\right)$, and $\mathcal{A}_{g_{k}, d_{k}, l}$ is the fine moduli space of polarized abelian $g_{k^{-}}$ folds of degree $d_{k}$ with level $l$-structure. We finish this section with two more results on $Y^{(k)}$ :

LEMMA 2.2. Let $V^{\delta}, h^{\delta}, \psi^{\delta}, K^{\delta}$, be as above. Let $F / E^{(0 \times 1)}$ be a field and let

$$
\xi: \operatorname{Spec} F \rightarrow K^{(0 \times 1)} M\left(G^{(0 \times 1)}, X^{(0 \times 1)}\right)
$$

be a point, let $Y_{\xi}^{(k)} / F$ be the abelian varieties which correspond to $g^{(k)}(\xi)$ via $(a)-(e)$ above (and leveled by the constraint $\eta^{(k)}\left(V_{\hat{\mathbb{Z}}}^{(k)}\right)=H_{1}\left(Y_{\xi}^{(k)}, \hat{\mathbb{Z}}\right)$ ). Then:

$$
H_{1}\left(Y_{\xi}^{(0)} \times_{F} F^{a c}, \mathbb{Z}_{\ell}\right)^{\otimes_{\mathcal{O}_{L_{\ell}}}{ }^{1-k}} \otimes_{\mathcal{O}_{L_{\ell}}} \bigwedge_{\mathcal{O}_{L_{\ell}}}^{k} H_{1}\left(Y_{\xi}^{(1)} \times_{F} F^{a c}, \mathbb{Z}_{\ell}\right)
$$

is isomorphic to

$$
H_{1}\left(Y_{\xi}^{(k)} \times_{F} F^{a c}, \mathbb{Z}_{\ell}\right)
$$

as $a \mathcal{O}_{L_{\ell}}\left[\mathrm{Gal}\left(F^{a c} / F\right)\right]$-module.

Proof. We can lift $\xi$ to a point $\xi^{\prime}:$ Spec $F^{a c} \rightarrow M\left(G^{(0 \times 1)}, X^{(0 \times 1)}\right)$, using the moduli interpretation of $M^{(0 \times 1)}$ (see section 4 below) we can find the associated tuple

$$
\left(Y_{\xi}^{(1)}, Y_{\xi}^{(0)}, \iota^{(1)}, \iota^{(0)}, \lambda^{(0 \times 1)}, \eta^{(1)}, \eta^{(0)}\right)
$$


where $\lambda^{(0 \times 1)}$ is a homogeneous polarization of $Y_{\xi}^{(0)} \times Y_{\xi}^{(1)}$ and $\eta^{(0)}, \eta^{(1)}$ are $L$-linear similitudes:

$$
\eta^{(1)}: V_{\mathbb{A}}^{(1)} \rightarrow H_{1}\left(Y_{\xi}^{(1)}, \mathbb{A}^{\infty}\right)
$$

and

$$
\eta^{(0)}: V_{\mathbb{A}^{\infty}}^{(0)} \rightarrow H_{1}\left(Y_{\xi}^{(0)}, \mathbb{A}^{\infty}\right)
$$

of which the multipliers agree. Now let $\tau$ be an element of $\operatorname{Gal}\left(F^{a c} / F\right)$, as $\xi^{\prime}$ and $\tau\left(\xi^{\prime}\right)$ have the same image in $K^{(0 \times 1)} M\left(G^{(0 \times 1)}, X^{(0 \times 1)}\right)$ one can find $\gamma=$ $\left(\gamma^{(0)}, \gamma^{(1)}\right) \in K^{(0 \times 1)}$ with $\tau\left(\xi^{\prime}\right)=\xi^{\prime} \cdot \gamma$. Due to the $G^{(0 \times 1)}\left(\mathbb{A}^{\infty}\right)$-equivariance of the $\operatorname{map} M\left(G^{(0 \times 1)}, X^{(0 \times 1)}\right) \rightarrow M\left(G^{(k)}, X^{(k)}\right)$ it follows that $\tau\left(g^{(k)}\left(\xi^{\prime}\right)\right)=g^{(k)}\left(\xi^{\prime}\right) \cdot g^{(k)}(\gamma)$. However, $\xi^{\prime} \cdot \gamma$ is by definition equal to:

$$
\left(Y_{\xi}^{(1)}, Y_{\xi}^{(0)}, \iota^{(1)}, \iota^{(0)}, \lambda^{(0 \times 1)}, \eta^{(1)} \circ \gamma^{(1)}, \eta^{(0)} \circ \gamma^{(0)}\right)
$$

and analogously for $g^{(k)}\left(\xi^{\prime}\right) \cdot g^{(k)}(\gamma)$. Now use the formula (2.4) and one is done.

COROLlaRY 2.3. If the assumptions are as above, then:

$$
H_{1}^{d R}\left(Y_{\xi}^{(0)} / F\right)^{\otimes_{F \otimes L} 1-k} \otimes_{F \otimes L} \bigwedge_{F \otimes L}^{k} H_{1}^{d R}\left(Y_{\xi}^{(1)} / F\right)
$$

is isomorphic to

$$
H_{1}^{d R}\left(Y_{\xi}^{(k)} / F\right)
$$

as $F \otimes L$-module with Hodge filtration and Gauss-Manin connection.

Proof. Without loss of generality one can assume that $F$ is a finitely generated extension of $E^{(0 \times 1)}$. We choose an embedding of $F$ into $\mathbb{C}$. By very construction of the map $g^{(k)}: K^{(0 \times 1)} M\left(G^{(0 \times 1)}, X^{(0 \times 1)}\right) \rightarrow K^{(k)} M\left(G^{(k)}, X^{(k)}\right)$ there are isomorphisms of Hodge structures with $\mathcal{O}_{L}$-operation:

$$
\begin{gathered}
H_{1}\left(Y_{\xi}^{(0)}(\mathbb{C}), \mathbb{Z}\right)^{\otimes_{\mathcal{O}_{L}} 1-k} \otimes_{\mathcal{O}_{L}} \bigwedge_{\mathcal{O}_{L}}^{k} H_{1}\left(Y_{\xi}^{(1)}(\mathbb{C}), \mathbb{Z}\right) \\
\left.t^{(k)}\right|_{1}\left(Y_{\xi}^{(k)}(\mathbb{C}), \mathbb{Z}\right)
\end{gathered}
$$

Let us denote by $t_{d R}^{(k)}$ and $t_{e t}^{(k)}$ the de Rham and étale realizations which are obtained from $t^{(k)}$ by composing with the comparison isomorphisms:

$$
H_{1}^{d R}\left(Y_{\xi}^{(k)} / \mathbb{C}\right) \cong H_{1}\left(Y_{\xi}^{(k)}(\mathbb{C}), \mathbb{C}\right)
$$

and

$$
H_{1}\left(Y_{\xi}^{(k)} \times_{F} F^{a c}, \hat{\mathbb{Z}}\right) \cong H_{1}\left(Y_{\xi}^{(k)}(\mathbb{C}), \hat{\mathbb{Z}}\right)
$$

The pairs $\left(t_{d R}^{(k)}, t_{e t}^{(k)}\right)$ are Hodge cycles (on the abelian variety $Y_{\xi}^{(0)^{\times_{F} k-1}} \times_{F} Y_{\xi}^{(1)} \times_{F}$ $\left.Y_{\xi}^{(k)}\right)$ in the sense of $[3$, Paragraph 2]. By [3, Theorem 2.11] these are absolute 
Hodge cycles (on the aforementioned product of abelian varieties over $F^{a c}$ ). By [3, Proposition 2.5] this implies that the de Rham components $t_{d R}^{(k)}$ are horizontal with respect to the Gauss-Manin connection, in particular they descend to isomorphisms over $F^{a c}$ :

$$
\begin{gathered}
H_{1}^{d R}\left(Y_{\xi}^{(0)} / F^{a c}\right)^{\otimes_{F}{ }^{a c} \otimes L}{ }^{1-k} \otimes_{F^{a c} \otimes L} \bigwedge_{F^{a c} \otimes L}^{k} H_{1}^{d R}\left(Y_{\xi}^{(1)} / F^{a c}\right) \\
\left.t_{d R}^{(k)}\right\rfloor_{1}^{d R}\left(Y_{\xi}^{(k)} / F^{a c}\right),
\end{gathered}
$$

according to $\left[3\right.$, Corollary 2.7]. Moreover, the subgroup of $\operatorname{Gal}\left(F^{a c} / F\right)$ fixing $t_{d R}^{(k)}$ is the same as the subgroup of $\mathrm{Gal}\left(F^{a c} / F\right)$ fixing $t_{e t}^{(k)}$, essentially because Galois conjugates of absolute Hodge cycles are again absolute Hodge cycles, [3, Proposition 2.9]. Lemma 2.2 finishes our proof as $t_{e t}^{(k)}$, is indeed rational over $F$, so that our horizontal map $t_{d R}^{(k)}$ descends to $F$ :

$$
\begin{gathered}
H_{1}^{d R}\left(Y_{\xi}^{(0)} / F\right)^{\otimes_{F \otimes L} 1-k} \otimes_{F \otimes L} \bigwedge_{F \otimes L}^{k} H_{1}^{d R}\left(Y_{\xi}^{(1)} / F\right) \\
\left.t_{d R}^{(k)}\right\rfloor \\
H_{1}^{d R}\left(Y_{\xi}^{(k)} / F\right)
\end{gathered}
$$

too.

REMARK 2.4. As we can identify $G^{(0)}$ with a subgroup of $G^{(1)}$, we can identify $G^{(0 \times 1)}$ with the product $G^{(0)} \times G$ where $G$ is the kernel of the map $g^{(0)}: G^{(0 \times 1)} \rightarrow G^{(0)}$. We even get a product of Shimura data $\left(G^{(0 \times 1)}, X^{(0 \times 1)}\right) \cong\left(G^{(0)}, X^{(0)}\right) \times(G, X)$, where $X$ is the projection of $X^{(0 \times 1)}$ onto the $G$-factor.

Notice however, that $(G, X)$ is not the type of Shimura datum, that can arise from any PEL-datum, nor is it of Hodge type. This is because its weight homomorphism is trivial. In fact one can think of $M_{\mathbb{C}}(G, X)$ as a moduli space for the Hodge structures $V^{(0)}{ }^{\otimes \mathcal{O}_{L}-1} \otimes_{\mathcal{O}_{L}} V^{(1)}$ which have weight zero.

\section{Deformations of ordinary points.}

3.1. Canonical Coordinates. In this subsection $L$ is a finite extension of $\mathbb{Q}_{p}$, let $\mathcal{O}_{L}$ be its ring of integers, $\mathfrak{p}$ its maximal ideal, and $\mathbb{F}=\mathcal{O}_{L} / \mathfrak{p}$ its residue field. We need some background material on Barsotti-Tate groups with $\mathcal{O}_{L}$-operation. Let $R$ be a noetherian local $\mathcal{O}_{L}$-algebra, complete with respect to the maximal ideal $\mathfrak{m}$. Assume that the residue field $k=R / \mathfrak{m}$ is an algebraically closed field extension of $\mathbb{F}$. Let $\mathcal{G}$ be a Barsotti-Tate group over $R$. Let $\iota: \mathcal{O}_{L} \rightarrow \operatorname{End}(\mathcal{G})$ be a homomorphism. The pair $(\mathcal{G}, \iota)$ is called a $\mathcal{O}_{L}$-Barsotti-Tate group, if $\mathcal{O}_{L}$ acts on Lie $\mathcal{G}$ by means of the structural morphism $\mathcal{O}_{L} \rightarrow R$, see [9], for the definition of Lie $\mathcal{G}$. A few numerical invariants are noteworthy: the $\mathcal{O}_{L}$-height ht ${ }_{\mathcal{O}_{L}} \mathcal{G}$ of $\mathcal{G}$ is defined by $|\mathcal{G}[\mathfrak{p}]|=\left|\mathcal{O}_{L} / \mathfrak{p}\right|^{\text {ht }{ }^{\mathcal{O}_{L}}}{ }^{\mathcal{G}}$, and the dimension of $\mathcal{G}$ is the rank of the projective module Lie $\mathcal{G}$. Every $\mathcal{O}_{L}$-Barsotti-Tate group sits in a unique exact sequence:

$$
0 \longrightarrow \mathcal{G}^{\circ} \longrightarrow \mathcal{G} \longrightarrow \mathcal{G}^{e t} \longrightarrow 0
$$

where $\mathcal{G}^{\circ}$ is connected and $\mathcal{G}^{\text {et }}$ is étale. One calls $\mathcal{G}$ ordinary if and only if $\operatorname{dim} \mathcal{G}=\operatorname{ht}_{\mathcal{O}_{L}} \mathcal{G}^{\circ}$, in general one has an inequality $\operatorname{dim} \mathcal{G} \leq \mathrm{ht}_{\mathcal{O}_{L}} \mathcal{G}^{\circ}$. It follows from the Dieudonné-Manin classification that over an algebraically closed field there is one and 
only one ordinary $\mathcal{O}_{L}$-Barsotti-Tate group of say dimension $a$ and $\mathcal{O}_{L}$-height $a+b$, cf. $[6,(29.8)]$. Also, by the rigidity of étale covers there is one and only one étale $\mathcal{O}_{L}$-Barsotti-Tate group of given $\mathcal{O}_{L}$-height over any $R$. In fact the same is true at the other extreme:

LEMMA 3.1. Let $R$ be as above and let a be an integer. The category of $\mathcal{O}_{L^{-}}$ Barsotti-Tate groups $\mathcal{G}$ over $R$ with $a=\operatorname{dim} \mathcal{G}=h_{\mathrm{O}_{L}} \mathcal{G}$, is equivalent to the category of $\mathcal{O}_{L}$-Barsotti-Tate groups $\mathcal{G}$ over $k=R / \mathfrak{m}$ with $a=\operatorname{dim} \mathcal{G}=\mathrm{ht}_{\mathcal{O}_{L}} \mathcal{G}$.

Proof. Let $R$ be artinian, let $I \subset R$ be an ideal of square zero, let us endow it with the trivial divided power structure. Consider a $\mathcal{O}_{L}$-Barsotti-Tate group $\mathcal{G}_{0}$ over $R_{0}=R / I$ with associated crystal $D\left(\mathcal{G}_{0}\right)$, see [9]. Notice that the value $D\left(\mathcal{G}_{0}\right)_{R}$ over $R$ is a free $R \otimes \mathcal{O}_{L}$-module of rank $a$. We have to show that $\mathcal{G}_{0}$ has a unique lift to a $\mathcal{O}_{L}$-Barsotti-Tate group $\mathcal{G}$ over $R$. So consider all sequences:

$$
0 \longrightarrow F_{i l}^{1} \longrightarrow D\left(\mathcal{G}_{0}\right)_{R} \longrightarrow \operatorname{Lie} \mathcal{G} \longrightarrow 0
$$

where the quotient Lie $\mathcal{G}$ is a free $R$-module of rank $a$ on which the $\mathcal{O}_{L}$ action factors through $\mathcal{O}_{L} \rightarrow R$. This last condition actually means that Lie $\mathcal{G}$ is a quotient of the $R$-module $R \otimes \mathcal{O}_{L} \mathcal{O}_{L}^{a}=R^{a}$. Due to rank reasons we then have equality. [

From this it follows easily that we have canonical coordinates for ordinary $\mathcal{O}_{L^{-}}$ Barsotti-Tate groups, we write $\Sigma / \mathcal{O}_{L}$ for the Lubin-Tate one, $\hat{\Sigma} / \mathcal{O}_{L}$ for its formal group, by $\hat{\Sigma}(R)$ we mean the set $\mathfrak{m}$ which is given a $\mathcal{O}_{L}$-module structure by the group law of $\hat{\Sigma}$.

LEMMA 3.2. Let $k=R / \mathfrak{m}$ be as above and let $\mathcal{G}_{0}$ be an ordinary $\mathcal{O}_{L}$-Barsotti-Tate group over $k / \mathbb{F}$. Consider the $\mathcal{O}_{L}$-modules:

$$
T^{\prime}=\operatorname{Hom}_{\mathcal{O}_{L}}\left(L / \mathcal{O}_{L}, \mathcal{G}_{0}\right)
$$

and

$$
T^{\prime \prime}=\operatorname{Hom}_{\mathcal{O}_{L}}\left(\Sigma \times \mathcal{O}_{L} k, \mathcal{G}_{0}\right) .
$$

There exists an equivalence of categories between lifts $\mathcal{G} / R$ of $\mathcal{G}_{0}$ and maps $\phi \in$ $\operatorname{Hom}_{\mathcal{O}_{L}}\left(T^{\prime}, T^{\prime \prime}\right) \otimes_{\mathcal{O}_{L}} \hat{\Sigma}(R)$, the equivalence being established by decreeing $\mathcal{G}$ to be the following push out:

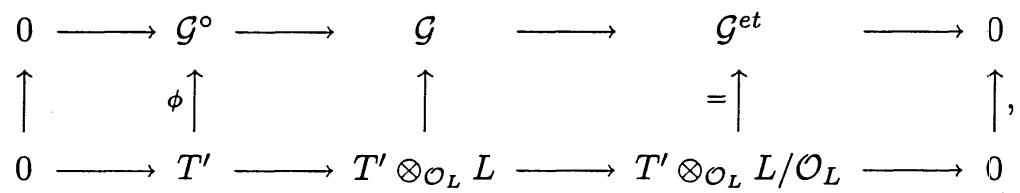

where $\mathcal{G}^{\circ}=T^{\prime \prime} \otimes_{\mathcal{O}_{L}} \Sigma \times_{\mathcal{O}_{L}} R$.

Proof. The proof is word for word the same as in [7, Paragraph 2], so we are brief. It is enough to consider lifts $\mathcal{G}$ over artinian $\mathcal{O}_{L}$-algebras $R$. According to lemma 3.1 the group $\mathcal{G}^{\circ}$ is canonically isomorphic to $T^{\prime \prime} \otimes_{\mathcal{O}_{L}} \Sigma \times_{\mathcal{O}_{L}} R$. Moreover, by the rigidity of quasi-isogenies the extension class of:

$$
0 \longrightarrow T^{\prime \prime} \otimes_{\mathcal{O}_{L}} \Sigma \times_{\mathcal{O}_{L}} R \longrightarrow \mathcal{G} \longrightarrow T^{\prime} \otimes_{\mathcal{O}_{L}} L / \mathcal{O}_{L} \longrightarrow 0
$$

is torsion, say killed by $p^{n}$. It follows that every element in

$$
\operatorname{Ext}_{\mathcal{O}_{L}}^{1}\left(T^{\prime} \otimes \mathcal{O}_{L} L / \mathcal{O}_{L}, T^{\prime \prime} \otimes_{\mathcal{O}_{L}} \Sigma \times_{\mathcal{O}_{L}} R\right)
$$


is induced from an element in

$$
\operatorname{Hom}_{\mathcal{O}_{L}}\left(T^{\prime} \otimes_{\mathcal{O}_{L}} p^{-n} \mathcal{O}_{L} / \mathcal{O}_{L}, T^{\prime \prime} \otimes_{\mathcal{O}_{L}} \Sigma \times \mathcal{O}_{L} R\right)
$$

which is unique as

$$
\operatorname{Hom}_{\mathcal{O}_{L}}\left(T^{\prime} \otimes \mathcal{O}_{L} L / \mathcal{O}_{L}, T^{\prime \prime} \otimes_{\mathcal{O}_{L}} \Sigma \times_{\mathcal{O}_{L}} R\right)=0
$$

In the sequel we will frequently use that the category of Barsotti-Tate groups over $R$ is equivalent to the category of projective systems of Barsotti-Tate groups over $R / M$ where $M$ runs through all $\mathfrak{m}$-primary ideals [9, Chapter II, Lemma(4.16)], in particular we can talk about the generic fibre of a deformation. In the ordinary case these can be obtained as follows:

LEMma 3.3. Let $\mathcal{G}_{0}$ be an ordinary $\mathcal{O}_{L}$-Barsotti-Tate group over $k / \mathbb{F}$, with dimension $a$ and $\mathcal{O}_{L}$-height $a+b$. Let $T^{\prime}, T^{\prime \prime}, R / \mathcal{O}_{L}$, and $\phi \in \operatorname{Hom}_{\mathcal{O}_{L}}\left(T^{\prime}, T^{\prime \prime}\right) \otimes_{\mathcal{O}_{L}} \hat{\Sigma}(R)$ with corresponding deformation $\mathcal{G} / R$ be as in lemma 3.2. Let $K / L$ be a field extension together with a $\mathcal{O}_{L}$-linear homomorphism $R \rightarrow K$. Pick $\mathcal{O}_{L}$-bases $e_{1}^{\prime}, \ldots, e_{b}^{\prime}$ of $T^{\prime}$, and $e_{1}^{\prime \prime}, \ldots, e_{a}^{\prime \prime}$ of $T^{\prime \prime}$, and let $\phi_{i, j} \in \hat{\Sigma}(R)$ be the entries of the matrix which represents $\phi$. Let further $\mathcal{G}_{i, j}$ be the $\mathcal{O}_{L}$-Barsotti-Tate group over $R$ defined by the push out:

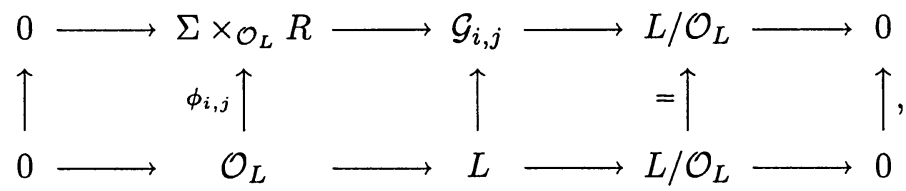

and let $c_{1}: \operatorname{Gal}\left(L^{a c} / L\right) \rightarrow \mathcal{O}_{L}^{\times}$be the character obtained from the Tate module $\mathcal{O}_{L}(1)$ of $\Sigma \times_{\mathcal{O}_{L}} L^{a c}$. Then the operation of $\operatorname{Gal}\left(K^{a c} / K\right)$ on the Tate module of $\mathcal{G} \times{ }_{R} K^{a c}$ is:

$$
\left(\begin{array}{cc}
c_{1} E_{a} & C \\
0 & E_{b}
\end{array}\right)
$$

where $C=\left(c_{i, j}\right)$ is the $a \times b$-matrix such that $c_{i, j}: \operatorname{Gal}\left(K^{a c} / K\right) \rightarrow \mathcal{O}_{L}(1)$ is any 1 -cocycle representing the continuous cohomology class in $H_{\text {cont }}^{1}\left(\mathrm{Gal}\left(K^{\text {ac }} / K, \mathcal{O}_{L}(1)\right)\right.$ which is obtained from the extension (3.1).

Proof. By addition of extension classes we may assume without loss of generality that at most one entry $\phi_{i_{0}, j_{0}}$ is non-zero. In this case $\mathcal{G}$ is isomorphic to $\mathcal{G}_{i_{0}, j_{0}} \oplus$ $\left(\Sigma \times \mathcal{O}_{L} R\right)^{a-1} \oplus\left(L / \mathcal{O}_{L}\right)^{b-1}$ and the assertion is obvious.

3.2. Canonical Lifts. We turn to consequences for abelian schemes with $\mathcal{O}_{L^{-}}$ operation, where $L$ is a $C M$-field. In analogy to subsection 3.1 we fix a rational prime $p$ and assume that, every prime of $L^{+}$over $p$ is split in $L$, hence there exists a set $\pi=\left\{\mathfrak{q}_{1}, \ldots, \mathfrak{q}_{m}\right\}$ of primes of $L$ over $p$, such that $\left\{\mathfrak{q}_{1}, \ldots, \mathfrak{q}_{m}, \mathfrak{q}_{1}^{*}, \ldots, \mathfrak{q}_{m}^{*}\right\}$ exhaust all the primes of $L$ over $p$, consequently we have a decomposition:

$$
\mathcal{O}_{L} \otimes \mathbb{Z}_{p} \cong \bigoplus_{i=1}^{m} \mathcal{O}_{L_{\mathfrak{q}_{i}}} \oplus \bigoplus_{i=1}^{m} \mathcal{O}_{L_{\mathfrak{q}_{i}^{*}}}
$$

We write $e_{1}, \ldots, e_{m}, e_{1}^{*}, \ldots, e_{m}^{*} \in \mathcal{O}_{L} \otimes \mathbb{Z}_{p}$ for the corresponding idempotents. Moreover for $i \in\{1, \ldots, r\}$, we want to fix embeddings $\sigma_{i}: L_{\mathfrak{q}_{i}} \rightarrow \mathbb{Q}_{p}^{a c}$, where we fix an 
algebraic closure $\mathbb{Q}_{p}^{a c}$ of $\mathbb{Q}_{p}$. We put $\mathcal{O}_{p}^{a c}$ for the integers in $\mathbb{Q}_{p}^{a c}$, and $\mathfrak{P} \mid p$ for the maximal ideal. Finally, $E$ is the field generated by the $\sigma_{i}\left(L_{\mathfrak{q}_{i}}\right)$ 's, with ring of integers $\mathcal{O}_{E}$, maximal ideal $\mathfrak{p}$, and residue field $\mathbb{F}_{\mathfrak{p}}$.

Let $S$ be a base scheme over $\mathcal{O}_{E}$ and let $A$ be an abelian scheme over $S$, and let $\lambda$ be a $p$-principal quasipolarization on $A / S$. We will say that some operation $\iota: \mathcal{O}_{L} \rightarrow \operatorname{End}(A)$, makes $(A, \lambda)$ into a $\mathcal{O}_{L}$-abelian scheme if and only if for every $i \in\{1, \ldots, r\}$ the induced operation on the projective $\mathcal{O}_{S}$-module $e_{i} \operatorname{Lie} A$ coincides with scalar multiplication by means of the map $\sigma_{i}: \mathcal{O}_{L_{\mathfrak{q}_{i}}} \rightarrow \mathcal{O}_{E} \rightarrow \Gamma(S, \mathcal{O})$, and if $e_{i} \operatorname{Lie} A=0$ for the remaining $i \in\{r+1, \ldots, m\}$. More specifically let $k$ be an algebraically closed field extension of $\mathbb{F}_{\mathfrak{p}}$. Then a $\mathcal{O}_{L}$-abelian scheme $(A, \lambda, \iota)$ over $k$ gives rise to $\mathcal{O}_{L_{\mathfrak{q}_{i}}}$-Barsotti-Tate groups $A\left[\mathfrak{q}_{i}^{\infty}\right]$. We will say that $(A, \lambda, \iota)$ is ordinary if this holds for all of the $A\left[\mathfrak{q}_{i}^{\infty}\right]$. In this case we can apply the Serre-Tate canonical coordinates to study deformations of the $\mathcal{O}_{L}$-abelian scheme $(A, \lambda, \iota)$ over any Spec $k \hookrightarrow \operatorname{Spec} R$, such that $R$ is a noetherian local $\mathcal{O}_{E}$-algebra that is complete with respect to its maximal ideal. In particular let us look at the unique unramified extension $B / E$ of complete discretely valued fields that has $k / \mathbb{F}_{\mathfrak{p}}$ as residue field extension. The lift $(\tilde{A}, \lambda, \iota)$ to $\mathcal{O}_{B}$ with the canonical coordinates of all the $\tilde{A}\left[\mathfrak{q}_{i}^{\infty}\right]$ the trivial ones is called the canonical lift. We have the following important fact:

Lemma 3.4. Fix $\pi=\left\{\mathfrak{q}_{1}, \ldots, \mathfrak{q}_{m}\right\}$ and $\sigma_{i}: L_{\mathfrak{q}_{i}} \rightarrow \mathbb{Q}_{p}^{a c}$ for $i \in\{1, \ldots, r\}$ as above. Let $(A, \lambda, \iota)$ be a $\mathcal{O}_{L}$-abelian variety over the algebraically closed field extension $k$ over $\mathbb{F}_{\mathfrak{p}}$. Let $(\tilde{A}, \lambda, \iota)$ be the canonical lift over $\mathcal{O}_{B}$. Then $\operatorname{End}_{\mathcal{O}_{L}}(\tilde{A})=\operatorname{End}_{\mathcal{O}_{L}}(A)$, as $\mathbb{Z}$ algebras with involution.

Proof. The proof in [9, Chapter V,Theorem(3.3)] translates word for word to our situation. [

\section{The integral model $\mathcal{M}^{(0 \times 1)}$.}

4.1. The moduli problem. Recall the two $C M$ traces $\Phi^{(0)}$ and $\Phi^{(n)}$ for $L$, as in section 2, and recall also our choice of $\left(V^{(1)}, V^{(0)}, \psi^{(1)}, \psi^{(0)}, h^{(1)}, h^{(0)}\right)$ giving rise to Shimura data $\left(G^{(0 \times 1)}, X^{(0 \times 1)}\right)$ and of the level $l \geq 3$. We fix a prime $\mathfrak{P} \mid p$ in $\mathbb{Q}^{a c}$, the algebraic closure of $\mathbb{Q}$ in $\mathbb{C}$, and assume $(\boldsymbol{})$ the following:

- the pairings $\psi^{(1)}$ and $\psi^{(0)}$ induce $\mathbb{Z}_{(p)}$-valued perfect pairings on $V_{\mathbb{Z}_{(p)}^{(1)}}$ and $V_{\mathbb{Z}_{(p)}^{(0)}}$

- $p$ is coprime to $l$,

- there exists a set $\pi$ of primes of $L$ over $p$ such that

$$
\left|\Phi^{(0)}\right|=\left\{\sigma: L \rightarrow \mathbb{Q}^{a c} \mid \sigma^{-1}(\mathfrak{P}) \in \pi^{*}\right\},
$$

- if $\left|\Phi^{(n)}\right|-\left|\Phi^{(0)}\right|=\left\{\sigma_{1}, \ldots, \sigma_{r}\right\}$ then $\mathfrak{q}_{1}=\sigma_{1}^{-1}(\mathfrak{P}), \ldots, \mathfrak{q}_{r}=\sigma_{r}^{-1}(\mathfrak{P})$ are pairwise distinct prime ideals of $L$.

We write $\left\{\mathfrak{q}_{i} \mid i=r+1, \ldots, m\right\}$ for the remaining primes in $\pi$. The primes in $\pi^{*}$ are nothing else then $\left\{\mathfrak{q}_{1}^{*}, \ldots, \mathfrak{q}_{m}^{*}\right\}$, consequently we have a decomposition as in (3.2) with corresponding idempotents $e_{1}, \ldots, e_{m}, e_{1}^{*}, \ldots, e_{m}^{*} \in \mathcal{O}_{L} \otimes \mathbb{Z}_{p}$. We write $E$ for the field generated by the $\sigma_{i}(L)$ 's, we write $\mathfrak{p}$ for the prime ideal induced in $E$ by $\mathfrak{P}$, and denote as usual by $\mathcal{O}_{E_{\mathfrak{p}}}$ the integers in the completion of $E$ at $\mathfrak{p}$, notice that $E$ contains the reflex field $E^{(0 \times 1)}$ and that $E_{\mathfrak{p}}$ may well be ramified over $E_{\mathfrak{p}}^{(0 \times 1)}$. The moduli interpretation for $M^{(0 \times 1)}$ that we will give is only defined over the extension $\mathcal{O}_{E_{\mathrm{p}}}$.

The first two - -conditions imply that the group $K^{(0 \times 1)}$, as introduced in (2.6), allows 
a factorization $K^{(0 \times 1)^{p}} K_{p}^{(0 \times 1)}$, where $K^{(0 \times 1)^{p}} \subset G^{(0 \times 1)}\left(\mathbb{A}^{\infty, p}\right)$ is compact open, and $K_{p}^{(0 \times 1)}=G_{\mathbb{Z}}^{(0 \times 1)}\left(\mathbb{Z}_{p}\right)$ looks like $\mathbb{Z}_{p}^{\times} \times \prod_{i=1}^{m} \mathcal{O}_{L_{\mathfrak{q}_{i}}}^{\times} \times \prod_{i=1}^{m} \mathrm{GL}\left(n, \mathcal{O}_{L_{\mathrm{q}_{i}}}\right)$. We begin by introducing a moduli space of abelian varieties. We need the following set valued functor, over a $\mathcal{O}_{E_{\mathrm{p}}}$-scheme $S$ its points consist of:

(a') $Y^{(1)}$ and $Y^{(0)}$, abelian schemes over $S$, up to $\mathbb{Z}_{(p)}$-isogeny,

(b') operations $\iota^{(1)}: \mathcal{O}_{L} \rightarrow \operatorname{End}\left(Y^{(1)}\right) \otimes \mathbb{Z}_{(p)}$ and $\iota^{(0)}: \mathcal{O}_{L} \rightarrow \operatorname{End}\left(Y^{(0)}\right) \otimes \mathbb{Z}_{(p)}$, such that for $k \in\{0,1\}$ the $\mathcal{O}_{S}$-modules $e_{i} \operatorname{Lie} Y^{(k)}$ are projective of rank 1 if $k=1$ and $i \in\{1, \ldots, r\}$ and of rank 0 otherwise, moreover in the former case the $\mathcal{O}_{L}$-operation on $e_{i} \operatorname{Lie} Y^{(1)}$ is given by $\sigma_{i}$.

(c') $\lambda^{(0 \times 1)}$, a homogeneous class of polarizations on $Y^{(0)} \times Y^{(1)}$ containing a representative of degree in $\mathbb{Z}_{(p)}^{\times}$,

(d') level- $K^{(0 \times 1)^{p}}$-structure $\bar{\eta}^{(0 \times 1)}$, i.e. for some choice of geometric point $\xi$ of $S$ one has a $\pi_{1}(S, \xi)$-invariant $K^{(1)^{p}}-\left(\right.$ resp. $K^{(0)^{p}}-$ ) class of $\mathcal{O}_{L} \otimes \mathbb{A}^{\infty, p}$-linear symplectic similitudes:

$$
\eta^{(1)}: V^{(1)} \otimes \mathbb{A}^{\infty, p} \rightarrow H_{1}\left(Y_{\xi}^{(1)}, \mathbb{A}^{\infty, p}\right)
$$

(resp.

$$
\eta^{(0)}: V^{(0)} \otimes \mathbb{A}^{\infty, p} \rightarrow H_{1}\left(Y_{\xi}^{(0)}, \mathbb{A}^{\infty, p}\right)
$$

), of which the multipliers agree.

The above functor is representable by a $\mathcal{O}_{E_{\mathrm{p}}}$-scheme $\mathcal{M}^{(0 \times 1)}$. The general fibre of it is canonically isomorphic to

$$
M^{(0 \times 1)}=\coprod_{i} K^{(0 \times 1)} M\left(G_{i}^{(0 \times 1)}, X^{(0 \times 1)}\right) \times_{E^{(0 \times 1)}} E_{\mathfrak{p}}
$$

where $i$ indexes all the locally trivial $G^{(0 \times 1)}$-torsors, and where $G_{i}^{(0 \times 1)}$ is the automorphism group of the $i$ th $G^{(0 \times 1)}$-torsor. It is clear that the constructions in section 2, can be applied to each of the Shimura varieties in (4.1) at a time, i.e. there are Shimura data $\left(G_{i}^{(k)}, X^{(k)}\right)$ corresponding to each of the locally trivial $G^{(0 \times 1)}$-torsors and there are maps

$$
g^{(k)}: K^{(0 \times 1)} M\left(G_{i}^{(0 \times 1)}, X^{(0 \times 1)}\right) \rightarrow K^{(k)} M\left(G_{i}^{(k)}, X^{(k)}\right)
$$

generalizing the morphism (2.7). In particular, by our conventions on the compact open subgroups $K^{(0 \times 1)}$ and $K^{(k)}$, we obtain homogeneously polarized abelian schemes with properties as expressed in lemma 2.2 over each of the Shimura varieties $K^{(0 \times 1)} M\left(G_{i}^{(0 \times 1)}, X^{(0 \times 1)}\right)$. We will continue to denote these by $Y^{(k)}$. The same remark applies to the induced classifying maps (2.8) which generalize to give maps:

$$
g^{(k)}: M^{(0 \times 1)} \rightarrow \mathcal{A}_{g_{k}, d_{k}, l} .
$$

Here is $g_{k}=\left[L^{+}: \mathbb{Q}\right]\left(\begin{array}{l}n \\ k\end{array}\right)$, and $d_{k}$ is the degree of some choice of an effective polarization within the homogeneous class $\lambda^{(k)}$.

LEMMA 4.1. If $\boldsymbol{\Lambda}$ holds, then $\mathcal{M}^{(0 \times 1)}$ is smooth over $\mathcal{O}_{E_{\mathfrak{p}}}$.

Proof. We use the concept of a local model $M^{l o c}$ over $\mathcal{O}_{E_{\mathrm{p}}}$, following the method of [10]. In the case at hand, the functor which $M^{l o c}$ represents, can be described as follows, over $S / \operatorname{Spec} \mathcal{O}_{E_{\mathfrak{p}}}$ the points consist of pairs $(t, \phi)$ where $t$ is a $\left(\bigoplus_{i=1}^{m} \mathcal{O}_{L_{\mathfrak{q}_{i}}}\right) \otimes_{\mathbb{Z}_{p}}$ $\mathcal{O}_{S}$ module, and $\phi:\left(\bigoplus_{i=1}^{m} \mathcal{O}_{L_{q_{i}}}\right)^{n} \otimes_{\mathbb{Z}_{p}} \mathcal{O}_{S} \rightarrow t$ is a $\left(\bigoplus_{i=1}^{m} \mathcal{O}_{L_{q_{i}}}\right) \otimes_{\mathbb{Z}_{p}} \mathcal{O}_{S}$-linear surjective map such that: 
- $e_{i} t$ is a line bundle on $S$, for all $i \in\{1, \ldots, r\}$, moreover $\mathcal{O}_{L_{\mathbf{q}_{i}}}$ acts on it by $\sigma_{i}$

- $e_{i} t=0$ for all $i \in\{r+1, \ldots, m\}$

One sees that $M^{l o c}$ is smooth, as the fibres are isomorphic to $\left(\mathbb{P}^{n-1}\right)^{\times r}$.

4.2. Stratification. Let $k$ be an algebraically closed field over $\mathbb{F}_{\mathfrak{p}}$ and let $\xi$ : Spec $k \rightarrow \mathcal{M}^{(0 \times 1)}$ be a point corresponding to data $\left(Y_{\xi}^{(1)}, Y_{\xi}^{(0)}, \ldots\right)$. If $i \in\{r+$ $1, \ldots, m\}$, then the $\mathcal{O}_{L_{\mathfrak{q}_{i}}}$-Barsotti-Tate group $Y_{\xi}^{(1)}\left[\mathfrak{q}_{i}^{\infty}\right]$ is étale, if $i \in\{1, \ldots, r\}$ it is one-dimensional, hence isomorphic to

$$
G_{1, n-f_{i}-1}^{\mathcal{O}_{L_{\mathbf{q}_{i}}}} \oplus\left(L_{\mathbf{q}_{i}} / \mathcal{O}_{L_{\mathbf{q}_{i}}}\right)^{f_{i}}
$$

for some $f_{i} \in\{0, \ldots, n-1\}$, here the notation is from $[6,(29.8)]$. The formal $\mathcal{O}_{L_{\mathbf{q}_{i}}}$-module is in this case isomorphic to

$$
G_{1, n-f_{i}-1}^{\mathcal{O}_{L_{\mathbf{q}_{i}}}}
$$

We call $\xi$ ordinary if $f_{1}=\cdots=f_{r}=n-1$, i.e. if and only if all the $\mathcal{O}_{L_{\mathfrak{q}_{i}}}$-BarsottiTate groups $Y_{\xi}^{(1)}\left[\mathfrak{q}_{i}^{\infty}\right]$ are ordinary in the sense of section 3 . The ordinary locus is Zariski open by [11]. We let $\mathcal{M}_{\text {ord }}^{(0 \times 1)}$ be the open subscheme of $\mathcal{M}^{(0 \times 1)}$ obtained by removing the non-ordinary locus in the special fibre.

5. An extension theorem. We continue the study of the abelian schemes $Y^{(k)}$, we begin with a lemma on the degree of $Y^{(k)}$ :

LEMMA 5.1. Let $\left(V^{(1)}, V^{(0)}, \psi^{(1)}, \psi^{(0)}\right)$ and corresponding $\left(V^{(k)}, \psi^{(k)}\right)$, be as in section 2. If $\psi^{(1)}$ and $\psi^{(0)}$ induce $\mathbb{Z}_{(p)}$-valued perfect pairings on $V_{\mathbb{Z}_{(p)}^{(1)}}$ and $V_{\mathbb{Z}_{(p)}}^{(0)}$, then $\psi^{(k)}$ induces a $\mathbb{Z}_{(p)}$-valued perfect pairing on $V_{\mathbb{Z}_{(p)}^{(k)}}$.

Proof. The data $\psi^{(k)}$ gives rise to a $\mathbb{Z}_{(p)^{-}}$-valued perfect pairing on the $\mathcal{O}_{L} \otimes \mathbb{Z}_{(p)^{-}}$ module $V_{\mathbb{Z}_{(p)}}^{(k)}$ if and only if

$$
\Psi^{(k)}: V_{\mathbb{Z}_{(p)}^{(k)}}^{(k)} V_{\mathbb{Z}_{(p)}^{(k)}}^{\left(k \mathcal{D}_{L}^{-1} \otimes \mathbb{Z}_{(p)}\right.}
$$

has this property, where $\mathcal{D}_{L}$ is the different of $\mathcal{O}_{L}$. If one has this for $k \in\{0,1\}$, then the same follows for the exterior pairing

$$
\bigwedge_{\mathcal{O}_{L}}^{k} V_{\mathbb{Z}_{(p)}^{(1)}} \times \bigwedge_{\mathcal{O}_{L}}^{k} V_{\mathbb{Z}_{(p)}^{(1)}}^{(1)} \rightarrow \mathcal{D}_{L}^{-k} \otimes \mathbb{Z}_{(p)},
$$

induced by $\Psi^{(1)}$, and for the $1-k$-fold self-product of $\Psi^{(0)}$ :

$$
V_{\mathbb{Z}_{(p)}^{(0)}}^{\otimes \mathcal{O}_{L} 1-k} \times V_{\mathbb{Z}_{(p)}^{(0)}}^{\otimes \mathcal{O}_{L}{ }^{1-k}} \rightarrow \mathcal{D}_{L}^{k-1} \otimes \mathbb{Z}_{(p)}
$$

By taking the product once more we obtain that

$$
\Psi^{(k)}: V_{\mathbb{Z}_{(p)}^{(k)}}^{(k)} V_{\mathbb{Z}_{(p)}}^{(k)} \rightarrow \mathcal{D}_{L}^{-1} \otimes \mathbb{Z}_{(p)}
$$

is perfect, which is what we wanted. 
5.1. Extension of $Y^{(k)}\left[\mathfrak{q}_{i}^{\infty}\right]$. With these gadgets we are now in a position to give a Serre-Tate analog of the tensor constructions of section 2. Let us start with an algebraically closed extension $k / \mathbb{F}_{\mathfrak{p}}$ and an ordinary $k$-valued point $\xi: \operatorname{Spec} k \rightarrow$ $\mathcal{M}_{\text {ord }}^{(0 \times 1)}$, that is represented by the tuple $\left(Y_{\xi}^{(1)}, Y_{\xi}^{(0)}, \iota^{(1)}, \iota^{(0)}, \lambda^{(0 \times 1)}, \ldots\right)$. Our aim in this subsection is to define a certain Barsotti-Tate group:

$$
\mathcal{G}^{(k)}=\bigoplus_{i=1}^{m} \mathcal{G}^{(k)}\left[\mathfrak{q}_{i}^{\infty}\right] \oplus \bigoplus_{i=1}^{m} \mathcal{G}^{(k)}\left[\mathfrak{q}_{i}^{* \infty}\right]
$$

on the universal deformation space $\operatorname{Defo}_{\xi}=\operatorname{Spec} R$ of $\xi$, in such a manner that the generic fibre of it matches our $Y^{(k)}\left[\mathfrak{q}_{i}^{\infty}\right]$. Here we use the usual algebraization results of polarized formal abelian schemes to freely switch between $\operatorname{Spec} R$ and $\operatorname{Spf} R$. Note that according to lemma 3.2 we have a canonical isomorphism

$$
\operatorname{Spf} R \cong \prod_{i=1}^{r} \operatorname{Hom}_{\mathcal{O}_{L_{\mathfrak{q}_{i}}}}\left(T_{i}^{(1)^{\prime}}, T_{i}^{(1)^{\prime \prime}}\right) \otimes_{\mathcal{O}_{L_{\mathfrak{q}_{i}}}} \hat{\Sigma}_{i} \times_{\mathcal{O}_{L_{\mathfrak{q}_{i}}}, \sigma_{i}} \mathcal{O}_{B}
$$

where $\mathcal{O}_{B} \subset B$ is again the ring of integers in the unique complete unramified extension $B$ of $E_{\mathfrak{p}}$ inducing the residue extension $k / \mathbb{F}_{\mathfrak{p}}$. It goes without saying that $\mathcal{G}^{(k)}\left[\mathfrak{q}_{i}^{* \infty}\right]$ will be the Serre dual of $\mathcal{G}^{(k)}\left[\mathfrak{q}_{i}^{\infty}\right]$. It is then meaningful to put:

$$
T_{i}^{(k)^{\prime}}=\operatorname{Hom}_{\mathcal{O}_{L_{\mathfrak{q}_{i}}}}\left(L_{\mathfrak{q}_{i}} / \mathcal{O}_{L_{\mathfrak{q}_{i}}}, Y^{(k)}\left[\mathfrak{q}_{i}^{\infty}\right]\right)
$$

and

$$
T_{i}^{(1)^{\prime \prime}}=\operatorname{Hom}_{\mathcal{O}_{L_{\mathfrak{q}_{i}}}}\left(\Sigma_{i} \times \mathcal{O}_{L_{\mathfrak{q}_{i}}}, \sigma_{i} k, Y^{(1)}\left[\mathfrak{q}_{i}^{\infty}\right]\right),
$$

for $i \in\{1, \ldots, m\}$, and $k \in\{0,1\}$, and further

$$
T_{i}^{(k)^{\prime}}=T_{i}^{(0)^{\prime}}{ }^{\otimes \mathcal{O}_{L_{q_{i}}}}{ }^{1-k} \otimes \mathcal{O}_{L_{q_{i}}} \bigwedge_{\mathcal{O}_{L_{q_{i}}}}^{k} T_{i}^{(1)^{\prime}}
$$

and

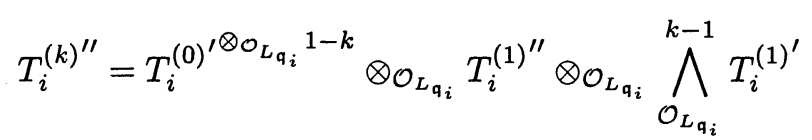

Let us also introduce a map:

$$
g_{c a n, i}^{(k)}: \operatorname{Hom}_{\mathcal{O}_{L_{\mathfrak{q}_{i}}}}\left(T_{i}^{(1)^{\prime}}, T_{i}^{(1)^{\prime \prime}}\right) \rightarrow \operatorname{Hom}_{\mathcal{O}_{L_{\mathfrak{q}_{i}}}}\left(T_{i}^{(k)^{\prime}}, T_{i}^{(k)^{\prime \prime}}\right)
$$

which is defined by contraction of elements, sending $\phi^{(1)}: T_{i}^{(1)^{\prime}} \rightarrow T_{i}^{(1)^{\prime \prime}}$ to the map $\phi^{(k)}: T_{i}^{(k)^{\prime}} \rightarrow T_{i}^{(k)^{\prime \prime}}$ defined by

$$
x_{0}^{1-k} x_{1} \wedge \cdots \wedge x_{k} \mapsto \sum_{\nu=1}^{k}(-1)^{\nu-1} x_{0}^{1-k} \phi^{(1)}\left(x_{\nu}\right) x_{1} \wedge \cdots \wedge x_{\nu-1} \wedge x_{\nu+1} \wedge \cdots \wedge x_{k}
$$

where $x_{0} \in T_{i}^{(0)^{\prime}}-\{0\}$ and $x_{1}, \ldots, x_{k} \in T_{i}^{(1)^{\prime}}$. Notice that $T_{i}^{(k)^{\prime \prime}}$ and $g_{c a n, i}^{(k)}$ are only non-zero if $T_{i}^{(1)^{\prime \prime}}$ is non-zero, which happens for all $i$ in $\{1, \ldots, r\}$. To define a $\mathcal{O}_{L_{\mathfrak{q}_{i}}}$-Barsotti-Tate group $\mathcal{G}^{(k)}\left[\mathfrak{q}_{i}^{\infty}\right]$ of $\mathcal{O}_{L_{\mathfrak{q}_{i}}}$-height $\left(\begin{array}{l}n \\ k\end{array}\right)$ over Defo $\xi$ we consider

$$
T_{i}^{(k)^{\prime \prime}} \otimes \mathcal{O}_{L_{\mathfrak{q}_{i}}} \Sigma_{i} \times \mathcal{O}_{L_{\mathbf{q}_{i}}}, \sigma_{i} k \oplus T_{i}^{(k)^{\prime}} \otimes \mathcal{O}_{L_{\mathbf{q}_{i}}} L_{\mathfrak{q}_{i}} / \mathcal{O}_{L_{\mathbf{q}_{i}}}
$$


and deform it by $g_{c a n, i}^{(k)}$. We can finish this subsection with the following two auxiliary lemmas:

LEMMA 5.2. Let $\pi_{1}$ be a profinite group. Let $\rho$ be a continuous representation thereof into the group $\mathrm{GL}(n, A)$, where $A$ is a ring, separated and complete with respect to the $M$-adic topology where $M$ is an ideal. Assume that $\rho$ has the form

$$
\left(\begin{array}{cccc}
c_{1} & c_{2} & \ldots & c_{n} \\
0 & 1 & \ldots & 0 \\
\vdots & \vdots & \ddots & \vdots \\
0 & 0 & \ldots & 1
\end{array}\right)
$$

where $c_{1}: \pi_{1} \rightarrow A^{\times}$is a continous character and where the maps $c_{2}, \ldots, c_{n}: \pi_{1} \rightarrow A$ are continous 1-cocycles of $\pi_{1}$ with coefficients in $c_{1}$ (i.e. representatives of elements in $\left.H_{\text {cont }}^{1}\left(\pi_{1}, c_{1}\right)\right)$. Then for every $k \in\{0, \ldots, n\}$ the representation $\bigwedge^{k} \rho: \pi_{1} \rightarrow$ $\mathrm{GL}\left(\left(\begin{array}{l}n \\ k\end{array}\right), A\right)$ has the form

$$
\left(\begin{array}{cc}
c_{1} E_{\left(\begin{array}{c}
n-1 \\
k-1
\end{array}\right)} & C \\
0 & E_{\left(\begin{array}{c}
n-1 \\
k
\end{array}\right)}
\end{array}\right)
$$

where the $\left(\begin{array}{c}n-1 \\ k-1\end{array}\right) \times\left(\begin{array}{c}n-1 \\ k\end{array}\right)$-matrix $C$ can be described as follows: the rows are indexed by the set of $k-1$-element subsets $I=\left\{i_{2}, \ldots, i_{k}\right\} \subset\{2, \ldots, n\}$, the columns are indexed by the set of $k$-element subsets $J=\left\{i_{1}, \ldots, i_{k}\right\} \subset\{2, \ldots, n\}$, and the entry in the $I$ 'th row and $J$ 'th column is equal to

$$
\begin{cases}(-1)^{\nu-1} c_{i_{\nu}} & I=J-\left\{i_{\nu}\right\} \\ 0 & \text { otherwise }\end{cases}
$$

Proof. Denote the standard basis of $A^{n}$ by $e_{1}, \ldots, e_{n}$ the subspace spanned by $e_{1} \wedge e_{i_{2}} \cdots \wedge e_{i_{k}}$ is $\wedge^{k} \rho$ invariant and the quotient carries the trivial action. This show that the diagonal blocks are as asserted. To check the cocycle matrix note that:

$$
\begin{aligned}
& \bigwedge^{k} \rho\left(e_{i_{1}} \wedge \cdots \wedge e_{i_{k}}\right)-e_{i_{1}} \wedge \cdots \wedge e_{i_{k}} \\
& =\sum_{\nu=1}^{k}(-1)^{\nu-1} c_{i_{\nu}} e_{1} \wedge e_{i_{2}} \cdots \wedge e_{i_{\nu-1}} \wedge e_{i_{\nu+1}} \wedge \cdots \wedge e_{i_{k}}
\end{aligned}
$$

LEMMA 5.3. Let the tuple $\left(Y_{\xi}^{(1)}, Y_{\xi}^{(0)}, \iota^{(1)}, \iota^{(0)}, \lambda^{(0 \times 1)}, \ldots\right)$ over $k / \mathbb{F}_{\mathfrak{p}}$ be as before, and let $\operatorname{Defo}_{\xi} \cong \operatorname{Spec} R$ be the universal deformation space. Let $\mathcal{G}^{(0)}, \mathcal{G}^{(1)}$, and $\mathcal{G}^{(k)}$ be the Barsotti-Tate groups over $R$ constructed above. Then for every $i \in$ $\{1, \ldots, m\}$ the (étale!) Barsotti-Tate group with $\mathcal{O}_{L_{\mathfrak{q}_{i}}}$-operation:

$$
\left(\mathcal{G}^{(0)}\left[\mathfrak{q}_{i}^{\infty}\right] \times_{R} R\left[\frac{1}{p}\right]\right)^{\otimes \mathcal{O}_{L_{\mathfrak{q}_{i}}} 1-k} \otimes \mathcal{O}_{L_{\mathfrak{q}_{i}}} \bigwedge_{\mathcal{O}_{L_{\mathfrak{q}_{i}}}}^{k}\left(\mathcal{G}^{(1)}\left[\mathfrak{q}_{i}^{\infty}\right] \times_{R} R\left[\frac{1}{p}\right]\right)
$$

is canonically isomorphic to $\mathcal{G}^{(k)}\left[\mathfrak{q}_{i}^{\infty}\right] \times_{R} R\left[\frac{1}{p}\right]$.

Proof. We may regard these objects over $R\left[\frac{1}{p}\right]$ as representations of $\pi_{1}\left(\operatorname{Spec} R\left[\frac{1}{p}\right]\right.$, Spec $\left.k\right)$, now use lemma 5.2 , and lemma 3.3 . 


\subsection{Extension of $Y^{(k)}$. We continue to assume that is valid:}

THEOREM 5.4. The abelian scheme $Y^{(k)}$ over $M^{(0 \times 1)}$ extends to an abelian scheme $\mathcal{Y}^{(k)}$ over the whole of $\mathcal{M}_{\text {ord }}^{(0 \times 1)}$. It inherits a $\mathcal{O}_{L}$-operation from the $\mathcal{O}_{L^{-}}$ operation $\iota^{(k)}$ on $Y^{(k)}$ in a unique way.

Proof. As $\mathcal{M}_{\text {ord }}^{(0 \times 1)}$ is disconnected we consider the connected components separetely, let $\mathcal{N}$ be one of them, it is an integral scheme, write $N$ for the generic fibre. We consider the morphism $g^{(k)}: N \rightarrow \mathcal{A}_{g_{k}, d_{k}, l}$, obtained from the abelian scheme $Y^{(k)}$ together with a choice of effective polarization in the homogeneous class $\lambda^{(k)}$, as we did in (2.8). Here note that we can choose the effective polarization in the class $\lambda^{(k)}$ to have a degree $d_{k}$ coprime to $p$, because of lemma 5.1 . Let $\mathcal{N}_{0}$ be the normalization of the schematic closure of the graph in $\mathcal{N} \times \mathcal{A}_{g_{k}, d_{k}, l}$, let $\chi$ be the projection from $\mathcal{N}_{0}$ to the $\mathcal{N}$-component. Let $\bar{N}_{0}, \bar{N}$, and $\bar{A}_{g_{k}, d_{k}, l}$, be the special fibres of $\mathcal{N}_{0}, \mathcal{N}$, and $\mathcal{A}_{g_{k}, d_{k}, l}$.

We want to prove that the fibres of $\chi$ are all 0-dimensional. By the semicontinuity of fibre dimensions it is enough to consider $\mathbb{F}_{\mathfrak{p}}^{a c}$-valued points $x_{0}$ of $\bar{N}_{0}$, lying over some $\mathbb{F}_{\mathfrak{p}}^{a c}$-valued point $(x, y)$ of $\bar{N} \times \bar{A}_{g_{k}, d_{k}, l}$. Write $R$ for the local ring of $\mathcal{O}_{E_{\mathfrak{p}}^{n r}} \times \mathcal{O}_{E_{\mathfrak{p}}} \mathcal{N}_{0}$ at the closed point $x_{0}$ and let $I \subset R$ be the stalk at $x_{0}$ of the ideal sheaf to the closed immersion

$$
\mathbb{F}_{\mathfrak{p}}^{a c} \times \bar{N} \bar{N}_{0} \hookrightarrow \mathcal{O}_{E_{\mathfrak{p}}^{n r}} \times \mathcal{O}_{E_{\mathfrak{p}}} \mathcal{N}_{0}
$$

Write $\hat{R}$ and $\hat{I}$ for their completions at the maximal ideal to $x_{0}$. Consider the commutative diagram:

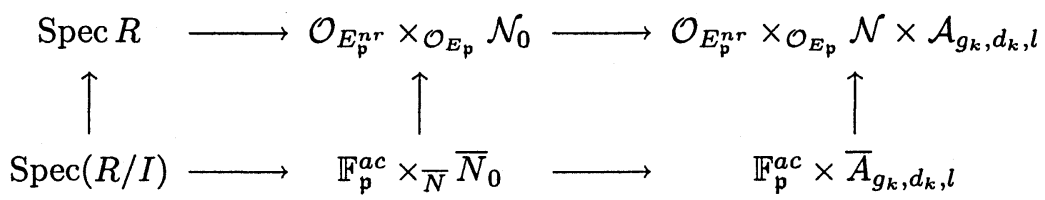

Over Spec $\hat{R}$ we have the pull-back of the universal abelian schemes $\mathcal{Y}^{(0)}, \mathcal{Y}^{(1)}$, and $\mathcal{Y}^{(k)}$. From the composition $\operatorname{Spec} \hat{R} \rightarrow \operatorname{Defo}_{x}$, we also have $\mathcal{O}_{L_{\mathbf{q}_{i}}}$-Barsotti-Tate groups $\mathcal{G}^{(k)}\left[\mathfrak{q}_{i}^{\infty}\right]$ according to subsection 5.1. By lemma 5.3 the generic fibre of $\mathcal{G}^{(k)}\left[\mathfrak{q}_{i}^{\infty}\right]$ agrees canonically with the generic fibre of $Y^{(k)}\left[\mathfrak{q}_{i}^{\infty}\right]$, according to $[15$, Theorem 4] $\mathcal{G}^{(k)}\left[\mathfrak{q}_{i}^{\infty}\right]$ agrees with $\mathcal{Y}^{(k)}\left[\mathfrak{q}_{i}^{\infty}\right]$. It follows that $\mathcal{Y}^{(k)}\left[\mathfrak{q}_{i}^{\infty}\right]$ is constant on $\operatorname{Spec}(\hat{R} / \hat{I})$ because $\mathcal{G}^{(k)}\left[\mathfrak{q}_{i}^{\infty}\right]$ is constant there. According to Serre-Tate, [9, Chapter V,Theorem 2.3] the polarized abelian scheme $\left(\mathcal{Y}^{(k)}, \lambda^{(k)}\right)$ is constant on $\operatorname{Spec}(\hat{R} / \hat{I})$, and on $\operatorname{Spec}(R / I)$ as well. This means that the natural map from the local ring $Q$ of $\mathbb{F}_{\mathfrak{p}}^{a c} \times \bar{A}_{g_{k}, d_{k}, l}$ at $y$ to $R / I$ factors through $y: Q \rightarrow \mathbb{F}_{\mathfrak{p}}^{a c}$. Now just notice that $R / I$ being the local ring of $\mathbb{F}_{\mathfrak{p}}^{a c} \times \bar{N}_{N} \bar{N}_{0}$ at $x_{0}$ is finite over $Q$, so that $R / I$ is finite over $\mathbb{F}_{\mathfrak{p}}^{a c}$.

We next prove that $\chi$ is proper, it certainly suffices to show that the schematic closure of $N$ is proper over $\mathcal{N}$, we check this by using the valuative criterion of properness, [4, Corollaire 7.3.10(ii)]. Let $F$ be the function field of $N$. Let $x: \operatorname{Spec} F \rightarrow N$ be the generic point, and let $g^{(k)} \circ x=y: \operatorname{Spec} F \rightarrow M^{(0 \times 1)}$ be the composition. Let $R$ be a discrete valuation ring of $F$, dominating some local ring of $\mathcal{O}_{\mathcal{N}}$. We write $\mathfrak{x}: \operatorname{Spec} R \rightarrow \mathcal{N}$ for the corresponding morphism, and $\mathcal{Y}_{\mathfrak{x}}^{(0)}$ and $\mathcal{Y}_{\mathfrak{x}}^{(1)}$, 
(resp. $Y_{x}^{(0)}$ and $Y_{x}^{(1)}$ ), for the pull backs of the universal abelian schemes over $\mathcal{M}^{(0 \times 1)}$ via $\mathfrak{x}$ (resp. via $x$ ). Choose any prime $\ell$ different from $p$. The $\ell$-adic Tate modules of $Y_{x}^{(0)}$ and $Y_{x}^{(1)}$ are unramified. Therefore, if $Y_{y}^{(k)}$ denotes the pull back of $Y^{(k)}$ to $\operatorname{Spec} F$, the $\ell$-adic Tate module of $Y_{y}^{(k)}$ is unramified as well, by lemma 2.2. Due to Néron-Ogg-Safarevic's criterion, [17, Theorem 1], there exists an abelian scheme $\mathcal{Y}_{\mathfrak{y}}^{(k)}$ over $R$ extending $Y_{y}^{(k)}$, moreover $\mathcal{Y}_{\mathfrak{y}}^{(k)}$ inherits a polarization of degree $d_{k}$ and a level $l$-structure from $Y_{y}^{(k)}$. The corresponding $R$-valued point in the moduli space $\mathcal{A}_{g_{k}, d_{k}, l}$ establishes a $R$-valued point $\mathfrak{y}$ in the schematic closure of $N$, which lies over $\mathfrak{x}$.

We conclude that $\chi$ is an isomorphism, by using the Main Theorem of Zariski [5, Corollaire 4.4.9].

The abelian schemes thus obtained have a $\mathcal{O}_{L}$-operation, for example because homomorphisms between abelian schemes over normal bases extend, [12, Chap.IX, Corollaire 1.4].

REMARK 5.5. The $\mathcal{Y}^{(k)}$ 's are $\mathcal{O}_{L}$-abelian schemes, because the structure of the $\mathcal{O}_{L}$-operation on the Lie algebra can be checked in the generic fibre.

Over algebraically closed fields of characteristic $p$ one can clarify how the canonical lift of $Y^{(1)}$ relates to the canonical lift of $Y^{(k)}$ :

LEMMA 5.6. Let $k$ be an algebraically closed field over $\mathbb{F}_{\mathfrak{p}}$. Let $\left(Y^{(1)}, Y^{(0)}\right.$, $\left.\iota^{(1)}, \iota^{(0)}, \lambda^{(0 \times 1)}, \bar{\eta}^{(0 \times 1)}\right)$ be a k-valued point of $\mathcal{M}_{\text {ord }}^{(0 \times 1)}$. Let $\left(\tilde{Y}^{(1)}, \tilde{Y}^{(0)}, \ldots\right)$ be the canonical lift over $\mathcal{O}_{B}$. Let $\mathfrak{x}: \operatorname{Spec} \mathcal{O}_{B} \rightarrow \mathcal{M}_{\text {ord }}^{(0 \times 1)}$ be the classifying morphism. Then the $\mathcal{O}_{L}$-abelian scheme $\mathcal{Y}_{\mathfrak{x}}^{(k)}$ over $\mathcal{O}_{B}$ is the canonical lift of its special fibre.

Proof. We only have to check that all the $\mathcal{O}_{L_{\mathfrak{q}_{i}}}$-Barsotti-Tate groups split, again by $[15$, Theorem 4] this follows if the Galois representation splits. This is clear, by lemma 2.2.

6. Endomorphism ring of $Y^{(k)}$. In [14] the endomorphism ring of the generic $Y^{(k)}$ is studied. We extend this study to the special fibre of $\mathcal{Y}^{(k)}$. We start with preliminary remarks on the Mumford-Tate group: Consider a $\mathbb{C}$-valued point $\left(Y_{\xi}^{(1)}\right.$, $\left.Y_{\xi}^{(0)}, \iota^{(1)}, \iota^{(0)}, \lambda^{(0 \times 1)}, \ldots\right)$ of $K^{(0 \times 1)} M\left(G^{(0 \times 1)}, X^{(0 \times 1)}\right)$, then

$$
V_{\mathbb{Q}}^{(1)}=H_{1}\left(Y^{(1)}(\mathbb{C}), \mathbb{Q}\right)
$$

is a Hodge structure with an operation of $L$ on it. Let us write $\mathcal{M} \mathcal{T} \subset \operatorname{GL}\left(V_{\mathbb{Q}}^{(1)} / L\right)$, for the smallest algebraic group over $L$ such that $\mathcal{M T} \times_{L, \sigma} \mathbb{C}$ contains for every embedding $\sigma: L \rightarrow \mathbb{C}$ the cocharacter $\mu_{\sigma}: \mathbb{G}_{m} \times \mathbb{C} \rightarrow \mathcal{M T} \times_{L, \sigma} \mathbb{C}$ given by

$$
\mu_{\sigma}(z): x \mapsto \begin{cases}z x & x \in V_{\sigma}^{(1)^{-1,0}} \\ x & x \in V_{\sigma}^{(1)^{0,-1}}\end{cases}
$$

the following is very well-known:

TheOREM 6.1. If $\operatorname{End}_{L}^{0}\left(Y_{\xi}^{(1)}\right)=L$ then the group $\mathcal{M T}$ to the Hodge structure with $L$-operation $V_{\mathbb{Q}}^{(1)}=H_{1}\left(Y_{\xi}^{(1)}, \mathbb{Q}\right)$ is the full linear group $\mathrm{GL}\left(V_{\mathbb{Q}}^{(1)} / L\right)$, in particular $\operatorname{End}_{L}^{0}\left(Y_{\xi}^{(k)}\right)=L$ for all $k$. 
Proof. We follow the ideas of [13, Theorem 3]. Let us remark that $V^{(1)}$ is semisimple as a representation of $\mathcal{M T}$. This is because any $\mathcal{M T}$-invariant subspace $W$ is a Hodge structure with $L$-operation. Then take the orthogonal complement $W^{*}$ with respect to $\psi^{(1)}$. This is again a Hodge structure with $L$-operation, and hence a $\mathcal{M T}$ subrepresentation complementary to $W$. So $\mathcal{M T}$ is reductive, as it has a faithful semisimple representation $\rho$, namely the natural action on the $L$ vector space $V_{\mathbb{Q}}^{(1)}$. A similar argument gives that $\operatorname{End}_{\mathcal{M} \mathcal{T}}(\rho)$ coincides with $\operatorname{End}_{L}\left(V^{(1)}\right)=L$.

Upon base change via some $\sigma: L \rightarrow \mathbb{C}$ we are in a position to apply [16, Proposition 5] to $\mathcal{M T} \times_{L, \sigma} \mathbb{C}$. Here note that we may choose $\sigma$ in the set $\left|\Phi^{(n)}\right|-\left|\Phi^{(0)}\right| \neq \emptyset$, so that the group $\mu_{\sigma}\left(\mathbb{G}_{m}\right)$ is contained in $\mathcal{M T} \times_{L, \sigma} \mathbb{C}$.

The assertion on $\operatorname{End}_{L}^{0}\left(Y_{\xi}^{(k)}\right)$ just follows as the $k$ th exterior power is an absolutely irreducible representation of $\mathrm{GL}\left(V_{\mathbb{Q}}^{(1)} / L\right)$.

Using canonical lifts we easily get an analog in positive characteristic:

Corollary 6.2. Assume that the -conditions of subsection 4.1 hold. Consider a point $\xi:$ Spec $k \rightarrow \mathcal{M}_{\text {ord }}^{(0 \times 1)}$, where $k$ is an algebraically closed field over $\mathbb{F}_{\mathfrak{p}}$. If $\operatorname{End}_{L}^{0}\left(\mathcal{Y}_{\xi}^{(1)}\right)=L$, then $\operatorname{End}_{L}^{0}\left(\mathcal{Y}_{\xi}^{(k)}\right)=L$, for all $k$.

Proof. Let $\mathfrak{x}: \operatorname{Spec} \mathcal{O}_{B} \rightarrow \mathcal{M}_{\text {ord }}^{(0 \times 1)}$ correspond to the canonical lift of $\left(\mathcal{Y}_{\xi}^{(1)}, \mathcal{Y}_{\xi}^{(0)}\right.$, $\left.\iota^{(1)}, \iota^{(0)}, \lambda^{(0 \times 1)}, \ldots\right)$, where $\mathcal{O}_{B}$ is as in subsection 3.2 . Choose an embedding $\mathcal{O}_{B} \hookrightarrow \mathbb{C}$. We have $\operatorname{End}_{L}^{0}\left(\mathcal{Y}_{\mathfrak{x}}^{(1)} \times_{\mathcal{O}_{B}} \mathbb{C}\right)=L$, because this algebra must be contained in $\operatorname{End}_{L}^{0}\left(\mathcal{Y}_{\xi}^{(1)}\right)=L$. By theorem 6.1 we infer $\operatorname{End}_{L}^{0}\left(\mathcal{Y}_{\mathfrak{x}}^{(k)} \times_{\mathcal{O}_{B}} \mathbb{C}\right)=L$, and therefore $\operatorname{End}_{L}^{0}\left(\mathcal{Y}_{\mathfrak{x}}^{(k)}\right)=L$. Now we apply lemmas 3.4 and 5.6.

\section{REFERENCES}

[1] P. Deligne, Travaux de Shimura, Séminaire Bourbaki Exposés pp. 382-399, Springer Lecture Notes in Math., 244, pp.123-165.

[2] P. Deligne, Variétés de Shimura: interprétation modulaire et techniques de construction de modèles canoniques, Proc. Symp. Pure. Math, 33 (1979), pp. 247-290.

[3] P. Deligne, Hodge cycles on abelian varieties, Hodge Cycles, Motives, and Shimura varieties, Springer Lecture Notes in Math., 900, pp. 9-100.

[4] A. GRothendieCK, Éléments de Géométrie Algébrique II, Étude globale élémentaire de quelques classes de morphismes.

[5] A. Grothendieck, Éléments de Géométrie Algébrique III, Étude Cohomologique des Faisceaux Cohérents (première partie).

[6] M. Hazewinkel, Formal Groups and Applications, Pure and Applied Mathematics 78.

[7] N. Katz, Serre-Tate Local Moduli, Surfaces Algébriques, Springer Lecture Notes in Math, 868, pp. 138-202.

[8] R. Kottwitz, Points on some Shimura varieties over finite fields, Journal of the AMS, 5 (1992), pp. 373-444.

[9] W. Messing, The Crystals Associated to Barsotti-Tate Groups: with Applications to Abelian Schemes, Springer Lecture Notes in Math. 264.

[10] M. RAPOPORT, T. ZINK, Period Spaces for p-divisible Groups, Annals of Mathematical Studies 141, Princeton University Press.

[11] M. RAPOPORT, M. RICHARTZ, On the classification and specialization of $F$-isocrystals with additional structure, Compos. Math., 103 (1996), pp. 153-181.

[12] M. RAYnAud, Faisceaux amples sur les schémas en groupes et les espaces homogènes, Springer Lecture Notes in Math. 119.

[13] K. RIBET, Hodge classes on certain types of abelian varieties, Amer. J. Math., 105 (1983), pp. 523-538. 
[14] I. SATAKe, Algebraic Structures of Symmetric Domains, Publications of the Mathematical Society of Japan 14, Iwanami Shoten 1980.

[15] J. TATE, $p$-divisible groups, Proceedings of a conference on local fields (1966).

[16] J.-P. SERre, Sur les groupes de Galois attachés aux groupes p-divisibles, Proceedings of a conference on local fields (1966).

[17] J.-P. Serre, J. TATE, Good reduction of Abelian varieties Ann. Math., 88 (1968), pp. $492-517$.

[18] A. VASIU, Integral canonical models of Shimura varieties of preabelian type, Asian J. Math., 3 (1999), pp. 401-518. 